\title{
Time-Varying Filters and Filter Banks: Some Basic Principles
}

\author{
See-May Phoong, Student Member, IEEE, and P. P. Vaidyanathan, Fellow, IEEE
}

\begin{abstract}
In this paper, we study the fundamentals of timevarying filter banks (TVFB). Using a polyphase approach to TVFB's, we are able to show some unusual properties that are not exhibited by the conventional LTI filter banks. For example, we can show that for a perfect reconstruction (PR) TVFB, the losslessness of analysis bank does not always imply that of the synthesis bank, and replacing the delay $z^{-1}$ in an implementation of a lossless linear time-variant (LTV) system with $z^{-L}$ for integer $L$ in general will result in a nonlossless system. Moreover, we show that interchanging the analysis and synthesis filters of a PR TVFB will usually destroy the PR property, and a PR TVFB in general will not generate a discrete-time basis for $l_{2}$.

Furthermore, we will show that we can characterize all TVFB's by characterizing multi-input multi-output (MIMO) LTV systems. A useful subclass of LTV systems, namely the lossless systems, will be discussed in detail. All lossless LTV systems are invertible. Moreover, the inverse is finite impulse response (FIR) if the original lossless system is FIR. Explicit construction of the inverses is given. However, unlike in the LTI case, we will show that the inverse system is not necessarily unique or invertible. In fact, the inverse of a lossless LTV system is not necessarily lossless. Depending on the invertibility of their inverses, the lossless systems are divided into two groups: i) invertible inverse lossless (IIL) systems and ii) noninvertible inverse lossless (NIL) systems. We will show that an NIL PR TVFB will only generate a discrete-time tight frame with unity frame bound. However if the PR FB is IIL, we will have an orthonormal basis for $l_{2}$. In a companion paper, some of these results are used to derive deeper properties of lossless TVFB including factorization theorems.
\end{abstract}

\section{INTRODUCTION}

$\mathbf{L}$ INEAR time-varying (LTV) filters have been considered in the past [1], [2]. In [1], the authors identified the constraints on the linear periodically time-varying (LPTV) infinite impulse response (IIR) filters. By using the derived constraints, stability was studied in detail and design technique was given. Review of LTV filters can be found in [1] and [2].

Recently, there has been considerable interest in both the theory and design of time-varying filter banks (TVFB's) [3]-[11]. In the applications of subband coding of speech and image signals, the advantages of TVFB's are demonstrated in [3]-[5]. It was shown that by using FB's with different

Manuscript received April 7, 1995; revised July 5, 1996. This work was supported by the NSF under Grant MIP 92-15785, by Tektronix, Inc., and by Rockwell International. The associate editor coordinating the review of this paper and approving it for publication was Dr. Bruce W. Suter.

S.-M. Phoong was with the Department of Electrical Engineering, California Institute of Technology, Pasadena, CA 91125 USA. He is now with the Department of Electrical and Electronic Engineering, Nanyang Technological University, Singapore.

P. P. Vaidyanathan is with the Department of Electrical Engineering, California Institute of Technology, Pasadena, CA 91125 USA (e-mail: ppvanath@sys.caltech.edu).

Publisher Item Identifier S 1053-587X(96)09046-0. responses on different regions (such as smooth areas and edge areas), low bit-rate compression with little ringing effect can be achieved. In this paper, we will focus mainly on the system-theoretic fundamentals of TVFB's whose analysis and synthesis filters are LTV. For the issues of design and applications, readers are referred to the above references.

In this paper, we present a theoretical study of TVFB's. Before we proceed to outline the paper, here is a brief review of recent literature on TVFB's as follows: these were first introduced in [6]. The authors used a time-domain approach to formulate the problem of switching between two perfect reconstruction (PR) FB's. To preserve the PR property during the transition, a number of synthesis banks is designed. The number of synthesis banks involved is usually quite large, and these transition synthesis banks are related to each other. In [3], arbitrary orthonormal tilings of the time-frequency plane were considered. The authors used an approach based on a matrix formulation of the two-channel FB's. Boundary and transition filters were constructed so that the orthogonality and PR were preserved when one switched from one paraunitary (PU) FB to another. Orthonormal TV wavelet bases were generated. In [7], the results in [3] were extended to the $M$ channel case. In [8], the authors considered the problem of switching between two LTI PU FB's, for which the LTI PU FB's were factorized into LTI degree one building blocks [12]. The authors combined the result of factorization of LTI PU FB's and the matrix approach in [7] to construct transition filters, so that PR was obtained. In all the approaches proposed in [3] and [6]-[8], there is a need for the construction of transition filters to preserve PR. The transition period puts a limit on how fast we can vary the filters. A different approach to TVFB's was given in [5] and [9]. The method was based on a generalization of the FIR lattice [12] to the time-varying case. In [5], the authors considered two-channel TVFB's. By cascading sections of FIR lattice with time-varying parameters, the authors showed that useful PR TVFB's can be obtained. The method was generalized to the $M$-channel case in [9], where the planar rotations [12] were made time varying. A cascade of these TV planar rotations and delays resulted in a lossless PR TVFB. The authors also provided a method to obtain a smooth transition when one switched from one PR FB to the other. In a companion paper [10], we will show that there are infinitely many lossless TVFB's that cannot be realized as a cascade of the time-varying FIR lattice. In [11], instead of redesigning filters during transition period, the authors designed a post filter such that the overall cascade system achieved nearly PR. 
In this paper, we will introduce an approach similar to the conventional polyphase method to study general properties of TVFB's. By using the proposed TV polyphase approach, we will address some basic theory for the general LTV filters and TVFB's. In particular, we are able to show the following differences between a conventional LTI FB and a TVFB.

1) In the LTI case, if a FB has PR, then the FB with analysis and synthesis filters interchanged has also the PR property [12, Problem 5.17]. In the LTV case, a similar statement is not true.

2) In the LTI PU case, both the analysis and synthesis banks are lossless [12]. In the LTV case, the losslessness of analysis bank does not imply that of the synthesis bank that yields PR.

3) If we replace the delay $z^{-1}$ in an implementation of a LTI PU system with $z^{-L}$ for some integer $L$, the system remains PU [12]. This is usually not true for a LTV lossless system.

4) In the LTI case, it is shown [13], [14] that a PR FB will generate a discrete-time Riesz basis for $l_{2}$ space. In the LTV case, a PR TVFB will only generate a frame for $l_{2}$. It becomes a basis only if the time-varying synthesis polyphase matrix is invertible.

There are many other properties of LTV filters and TVFB's which cannot be expected from the LTI cases. In order to show the differences between the LTV filters and the LTI filters, we discuss a single-input single-output (SISO) LTV system (which corresponds to an one-channel FB) in the following subsection.

\section{A. A Simple Time-Varying System}

Example 1.1: Consider the LTV system $\mathcal{H}$ given by $y(n)=$ $h_{0}(n) x(n)+h_{1}(n) x(n-1)$, where the coefficients are

$$
\begin{aligned}
& h_{0}(n)= \begin{cases}1, & \text { for } n<0 \\
0, & \text { for } n \geq 0,\end{cases} \\
& h_{1}(n)= \begin{cases}0, & \text { for } n \leq 0 \\
1, & \text { for } n>0 .\end{cases}
\end{aligned}
$$

One can verify that the output of the system is

$$
y(n)= \begin{cases}x(n), & \text { for } n<0 \\ 0, & \text { for } n=0 \\ x(n-1), & \text { for } n>0\end{cases}
$$

The above system has an inverse $\mathcal{G}$ given as $\hat{x}(n)=$ $g_{0}(n) y(n)+g_{-1}(n+1) y(n+1)$, where the coefficients $g_{0}(n)=h_{0}(n)$ and $g_{-1}(n)=h_{1}(n)$. The output of the inverse system is

$$
\hat{x}(n)= \begin{cases}y(n), & \text { for } n<0 \\ y(n+1), & \text { for } n \geq 0\end{cases}
$$

We see that $\hat{x}(n)=x(n)$ for all $n$. From this example, we can observe the following.

1) Losslessness: From (1.2), it is clear that $\sum_{n}|y(n)|^{2}=$ $\sum_{n}|x(n)|^{2}$, so the system $\mathcal{H}$ is lossless. In general, given an LTV system, it is difficult to test the losslessness by computing the output energy. In Section V, we will show how to characterize lossless systems in terms of their impulse responses.
2) Nontrivial SISO Lossless Systems: The system $\mathcal{H}$ has all the coefficients $h_{i}(n)$ equal to zero at $n=0$ and yet the system is lossless. This is not possible in LTI case. In the LTI case, the only scalar lossless system is the trivial system of the form $e^{j \theta} z^{-L}$. For the LTV case, there exist quite nontrivial scalar lossless FIR systems as we will explain in Section III and demonstrate in Section VI.

3) Existence and Uniqueness of Inverse: In the above example, it can be shown that for any choice of the constant $c$, the system described by $\hat{x}(n)=\left[h_{0}(n)+c \delta(n)\right] y(n)+$ $h_{1}(n+1) y(n+1)$ is an inverse for the lossless system of (1.2). Therefore, the inverse of a LTV lossless system is, in general, not unique. In Section $\mathrm{V}$, we will show that a lossless system is always invertible. The conditions for unique invertibility of lossless systems are studied in detail.

4) Noninvertibility and Nonlosslessness of the Inverse: It is clear from (1.3) that the inverse system $\mathcal{G}$ is not invertible because the sample $y(0)$ is lost and can never be recovered. In fact, the inverse system $\mathcal{G}$ is not lossless! This situation is different from the LTI case where the inverse of a lossless system is also invertible and lossless. In Section V, we will study the conditions under which the inverse of an LTV lossless system is invertible and lossless.

\section{B. Notations, Definitions, and Main Results}

Boldfaced letters are used to denote matrices and vectors. The complex-conjugation and transpose are denoted, respectively, by ${ }^{*}$ and ${ }^{T}$. The matrix $\mathrm{U}^{\dagger}(n)$ represents the transpose complex-conjugation of $\mathbf{U}(n)$. The coefficients of a LTV multi-input multi-output (MMMO) filter are denoted by matrices $e_{k}(n)$, where $n$ is the time index and $k$ is the coefficient index. All of the MIMO systems considered in this paper have $M$ inputs and $M$ outputs, therefore $\mathrm{e}_{k}(n)$ are $M \times M$ matrices. The calligraphic symbols such as $\mathcal{H}, \mathcal{G}$ in Example 1.1 are used to denote LTV systems. Given two systems $\mathcal{H}_{1}$ and $\mathcal{H}_{2}$, a cascade of $\mathcal{H}_{1}$ followed by $\mathcal{H}_{2}$ is denoted by $\mathcal{H}_{2} \mathcal{H}_{1}$. The output of a system $\mathcal{H}$ corresponding to the input $\mathrm{x}(n)$ is expressed as $\mathrm{y}(n)=\mathcal{H} \mathrm{x}(n)$, where both $\mathrm{y}(n)$ and $\mathrm{x}(n)$ are $M \times 1$ column vectors. All the TVFB's considered in this paper are uniform maximally decimated TVFB's, i.e., the number of channels is equal to the decimation ratio.

1) Definitions and Acronyms:

1) Inner Product: The inner product of two scalar sequences is defined as $\left\langle x_{1}(n), x_{2}(n)\right\rangle=$ $\sum_{n} x_{1}(n) x_{2}^{*}(n)$. For two vector sequences, their inner product is defined as $\left\langle\mathrm{x}_{1}(n), \mathrm{x}_{2}(n)\right\rangle=$ $\sum_{n} \mathrm{x}_{2}^{\dagger}(\dot{n}) \mathrm{x}_{1}(n)$

2) $l_{2}$-Norm and $l_{2}(M)$ Space: For a vector sequence $\mathrm{x}(n)$, its $l_{2}$-norm is defined as $\|\mathbf{x}(n)\|=\left[\sum_{n} \mathbf{x}^{\dagger}(n) \mathbf{x}(n)\right]^{1 / 2}$. The space of all finite norm $M$-dimensional vector sequences is denoted by $l_{2}(M)$. The space of all finite norm scalar sequences is simply represented by $l_{2}$.

3) Lossless System: A system is lossless if it is stable and preserves input/output energy, i.e., $\|\mathbf{y}(n)\|^{2}=\|\mathbf{x}(n)\|^{2}$. 
4) Passive System: A system is passive if it is stable and its output energy can never be greater than its input energy, i.e., $\|\mathbf{y}(n)\|^{2} \leq\|\mathbf{x}(n)\|^{2}$. Note that a lossless system is also passive.

5) Inverse System: A system $\mathcal{G}$ is said to be the inverse of a system $\mathcal{H}$ if the cascade of $\mathcal{H}$ followed by $\mathcal{G}$ is the identity system $\mathcal{I}$. The fact that $\mathcal{G}$ is an inverse of $\mathcal{H}$ is denoted by $\mathcal{G H}=\mathcal{I}$. In general, $\mathcal{G H}=\mathcal{I}$ does not imply $\mathcal{H G}=\mathcal{I}$, even for the case of scalar LTV systems (see Example 1.1).

6) Invertible/Noninvertible Inverse Lossless System: Lossless systems with invertible inverses are called invertible inverse lossless (IIL) systems. Lossless systems with noninvertible inverses are called noninvertible inverse lossless (NIL) systems. Note that there are no time-invariant NIL systems.

7) Order: Given a causal LTV filter $y(n)=$ $\sum_{k=0}^{N(n)} h_{k}(n) x(n-k)$, the order of the filter at time $n$ is the largest integer $k$ such that $h_{k}(n)$ is nonzero and this time-dependent integer is denoted by $N(n)$. If there is an integer $N<\infty$ such that $N(n) \leq N$ for all $n$, then we say the filter is FIR.

2) Main Results and Outline of the Paper: In Sections II-IV; we will present results for general LTV filters and filter banks, while in Sections V-VII, we will focus mainly on the class of lossless LTV filters and filter banks.

1) In Section II, we will first review some basics for LTV filters and introduce two direct form structures that will be used throughout the paper. Then a transform domain description for LTV filters will be defined and studied.

2) By using the transform domain description, we define the polyphase representations of LTV filters in Section III. We will show that noble identities similar to the LTI case hold for LTV systems. Utilizing the noble identities, we derive the efficient implementations for decimation filters and interpolation filters in the LTV case. The block implementation of an LTV filter is considered.

3) In Section IV, we will utilize the proposed polyphase representation to study some basic properties of TVFB, such as PR condition, interchangability of the analysis and synthesis filters, application to PR transmultiplexers, etc. Furthermore, we will show that we can characterize all TVFB's by characterizing all MIMO LTV systems.

4) In Section V, lossless LTV filters and filter banks will be considered. We will give both the impulse response and the transform domain characterizations of a lossless LTV system. All lossless FIR LTV systems are shown to be invertible, and their inverses are also FIR. Explicit formula for the inverse will be given. We will also discuss in detail some subtle properties of the inverse of lossless systems, such as uniqueness, invertibility, losslessness, etc. Furthermore, we will show that there will always exist a unique passive inverse for a lossless LTV system, and this passive inverse can be easily constructed.

5) In Section VI, some lossless LTV filter and filter bank examples will be provided to demonstrate the theory.

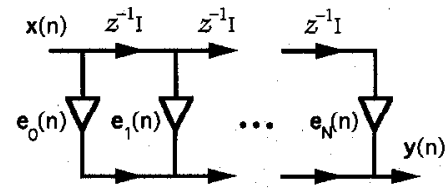

Fig. 1. Direct form A implementation of a Nth-order LTV filter.

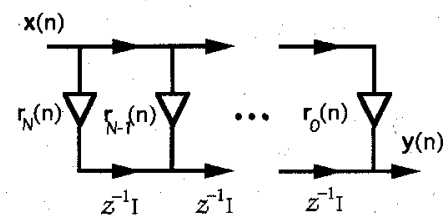

Fig. 2. Direct form B implementation of a $N$ th-order LTV filter.

6) In Section VII, a time-varying vector space approach to PR TVFB's is given. This can be viewed as a generalization of the approach proposed in [14]. By using this TV vector space approach, we will derive the orthonormality and biorthogonality conditions on the analysis and synthesis TV filters of TVFB's whose synthesis systems are also invertible. Furthermore, we will show that in general a PR TVFB only gives rise to a discrete-time frame for $l_{2}$ space. In the case of a NIL TVFB, it generates a tight frame with unity frame bound. In the case of IIL TVFB's, we can show that the synthesis (or the analysis) functions form an orthonormal basis for $l_{2}$.

\section{DiRECT Form StRUCTURES AND TRANSFORM DOMAIN DESCRIPTION OF LTV FILTERS}

A review of notations and different possible representations of LTV filters is given in [1] and [2]. Each of the representations is equivalent, and their relation to each other is explained in [1]. In this paper, we are going to use only two of the representations, which correspond to two different direct form implementations. In the following, we first study the two direct form implementations needed, and then define a transform description for each of the two representations.

\section{A. Direct Form A and B Implementations of LTV Filters}

Consider Figs. 1 and 2, where two different structures to implement an $N$ th-order MIMO causal LTV filter are shown. In the LTI case, these two structures are the same. In the LTV case, there is a simple one to one correspondence between these two structures. We will call the structures in Figs. 1 and 2 , respectively, the direct form A and B implementations. Their system equations can be, respectively, expressed as

$$
\begin{aligned}
\mathbf{y}(n)= & \mathbf{e}_{0}(n) \mathbf{x}(n)+\mathbf{e}_{1}(n) \mathbf{x}(n-1) \\
& +\cdots+\mathbf{e}_{N}(n) \mathbf{x}(n-N) \\
\mathbf{y}(n)= & \mathbf{r}_{0}(n) \mathbf{x}(n)+\mathbf{r}_{1}(n-1) \mathbf{x}(n-1) \\
& +\cdots+\mathbf{r}_{N}(n-N) \mathbf{x}(n-N)
\end{aligned}
$$

where the coefficients $\mathbf{e}_{k}(n)$ and $\mathbf{r}_{k}(n)$ are $M \times M$ matrices. From the above two equations, it is clear that if $\mathbf{r}_{k}(n-k)=$ $\mathbf{e}_{k}(n)$, the two filters in Figs. 1 and 2 are the same. We define 


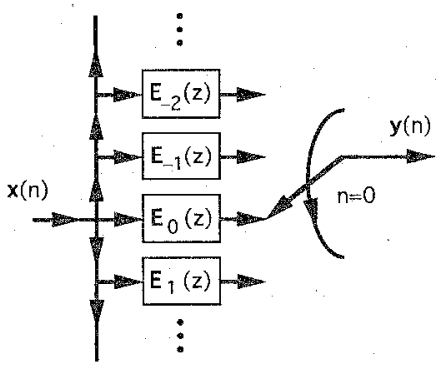

Fig. 3. Interpretation of direct form $\mathrm{A}$ in Fig. 1 by using a commutator switch and the LTI filters $E_{i}(z)$ defined in (2.3).

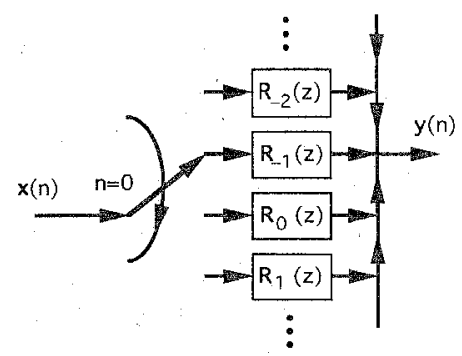

Fig. 4. Interpretation of direct form B in Fig. 2 by using a commutator switch and the LTI filters $R_{i}(z)$ defined in $(2.3)$.

the $z$-transform of the frozen systems in Figs. 1 and 2 at time $n$, respectively, as

$$
\begin{aligned}
& \mathbf{E}_{n}(z)=\sum_{k} \mathbf{e}_{k}(n) z^{-\dot{k}} \\
& \mathbf{R}_{n}(z)=\sum_{k} \mathbf{r}_{k}(n) z^{-k}
\end{aligned}
$$

where $\mathbf{E}_{n}(z)$ and $\mathbf{R}_{n}(z)$ are both LTI filters. It is shown in [1] that we can implement the LTV filters by using the LTI filters in (2.3) and commutators as shown in Figs. 3 and 4. In the case of LPTV filters with period $M$, we have $\mathbf{E}_{n+M}(z)=\mathbf{E}_{n}(z)$ and $\mathbf{R}_{n+M}(z)=\mathbf{R}_{n}(z)$. Figs. 3 and 4 , respectively, reduce to the LPTV systems given in Fig. 3(a) and (b) of [1]. From Fig. 3, it is clear that it is most convenient to implement a LTV decimation filter by using the direct form A structure. In a decimation filter, the output of the LTV filter is decimated by a factor of $M$, in this case, we need only to implement the subset $\mathbf{E}_{M n}(z)$. Similarly, in the case of interpolation filters, the direct form $\mathrm{B}$ is the most convenient because the input $\mathrm{x}(n)=0$ for $n \neq$ multiple of $M$, and we need only to implement $\mathbf{R}_{M n}(z)$. Even though both of the direct forms in Figs. 1 and 2 are equivalent, we will choose direct form $\mathrm{A}$ for decimation filters and direct form B for interpolation filters throughout the paper. The advantages of making such a choice will become clear as we go through the presentation.

\section{B. Transform Domain Representations of LTV Filters}

In the LTI case, polyphase representations are very useful tools in both the theory and design of multirate filter banks [12]. In the LTV case, since the conventional $z$-transform is undefined, we cannot apply the traditional polyphase definitions. We need to define a transform domain description for LTV filters similar to the conventional $z$-transform for

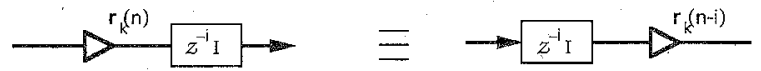

(a)

(b)

Fig. 5. Rule for interchanging a delay and a time-varying multiplier.

LTI filters. First, let us define the delay operator $\mathcal{Z}^{-1}$ as the following: i) $\mathcal{Z}^{-i} \mathrm{x}(n)=\mathrm{x}(n-i)$ for signal $\mathbf{x}(n)$; ii) $\mathcal{Z}^{-i_{0}} \mathcal{Z}^{-i_{1}}=\mathcal{Z}^{-i_{0}-i_{1}}$. The rule for interchanging a timedependent multiplier and the delay operator is described as $\mathcal{Z}^{-i} \mathbf{r}_{k}(n)=\mathbf{r}_{k}(n-i) \mathcal{Z}^{-i}$, as shown in Fig. 5. Note that the delay operator $\mathcal{Z}^{-1}$ is different from the $z$-transform; it does not commute with a multiplier unless the multiplier is time independent. Therefore the calligraphic symbol $\mathcal{Z}^{-1}$ is used for the delay operator to remind the readers of their difference from $Z^{-1}$. With the above delay operator, we can define the TV transform domain descriptions as

$$
\begin{aligned}
& \mathbf{E}(n, \mathcal{Z})=\sum_{k} \mathrm{e}_{k}(n) \mathcal{Z}^{-k} \\
& \mathbf{R}(\mathcal{Z}, n)=\sum_{k} \mathcal{Z}^{-k} \mathbf{r}_{k}(n)
\end{aligned}
$$

(for direct form B; Fig. 2).

Readers should note the use of $(n, \mathcal{Z})$ in (2.4) corresponding to direct form A in Fig. 1, and $(\mathcal{Z}, n)$ in (2.5) corresponding to direct form B in Fig. 2. By using the above TV transform domain descriptions, the output of the LTV filters in Figs. 1 and 2 can be written, respectively, as

$$
\begin{aligned}
\mathbf{y}(n) & =\mathbf{E}(n, \mathcal{Z}) \mathbf{x}(n)=\sum_{k} \mathbf{e}_{k}(n) \mathcal{Z}^{-k} \mathbf{x}(n) \\
& =\sum_{k} \mathbf{e}_{k}(n) \mathbf{x}(n-k) \\
\mathbf{y}(n) & =\mathbf{R}(\mathcal{Z}, n) \mathbf{x}(n)=\sum_{k} \mathcal{Z}^{-k} \mathbf{r}_{k}(n) \mathbf{x}(n) \\
& =\sum_{k} \mathbf{r}_{k}(n-k) \mathbf{x}(n-k) .
\end{aligned}
$$

In the transform domain, the rule of interchanging delays and LTV systems in Fig. 5 reduces to the following: i) $\mathcal{Z}^{-i} \mathbf{E}(n, \mathcal{Z})=\mathbb{E}(n-i, \mathcal{Z}) \mathcal{Z}^{-i}$ and ii) $\mathcal{Z}^{-i} \mathbf{R}(\mathcal{Z}, n)=$ $\mathbf{R}(\mathcal{Z}, n-i) \mathcal{Z}^{-i}$. The cascade of two LTV filters, $\mathbf{E}^{(0)}(n, \mathcal{Z})$ followed by $\mathbf{E}^{(1)}(n, \mathcal{Z})$, can be described as

$$
\mathbf{E}^{(1)}(n, \mathcal{Z}) \mathbf{E}^{(0)}(n, \mathcal{Z})=\sum_{k, l} \mathbf{e}_{k}^{(1)}(n) \mathrm{e}_{l}^{(0)}(n-k) \mathcal{Z}^{-k-l}
$$

where $\mathrm{e}_{k}^{(i)}(n)$ is the $k$ th coefficient of the LTV filter $\mathbf{E}^{(i)}(n, \mathcal{Z})$. Notice that, in general, $\mathbf{E}^{(1)}(n, \mathcal{Z}) \mathbf{E}^{(0)}(n, \mathcal{Z}) \neq$ $\mathbf{E}^{(0)}(n, \mathcal{Z}) \mathbb{E}^{(1)}(n, \mathcal{Z})$, even for the simplest case of scalar LTV filters.

\section{Polyphase Representations, Multirate IDENTITIES, AND BLOCK IMPLEMENTATIONS}

\section{A. Polyphase Representations}

With the transform domain description defined in the previous section, we are now ready to define the polyphase 
representations of LTV filters. Similar to the LTI case, we have two types of polyphase representations for both of the LTV filters in Figs. 1 and 2. First, consider Fig. 1 (the corresponding transform domain description is given in (2.4)). With respect to any positive integer $M$, the type 1 polyphase representation of the systems $\mathbf{E}(n, \mathcal{Z})$ can be defined as

$$
\begin{aligned}
\mathbf{E}(n, \mathcal{Z}) & =\sum_{i=0}^{M-1}\left[\sum_{k} \mathbf{e}_{k M+i}(n) \mathcal{Z}^{-k M}\right] \mathcal{Z}^{-i} \\
& =\sum_{i=0}^{M-1} \mathbf{E}_{i}\left(n, \mathcal{Z}^{M}\right) \mathcal{Z}^{-i}
\end{aligned}
$$

where $\mathbf{E}_{i}(n, \mathcal{Z})$ is called the $i$ th polyphase component of the type 1 representation at time $n$. Similarly, for the direct form B in Fig. 2 (the corresponding transform domain description is given in (2.5)), we can define the type 2 polyphase representation as

$$
\begin{aligned}
\mathbf{R}(\mathcal{Z}, n) & =\sum_{i=0}^{M-1} \mathcal{Z}^{i}\left[\sum_{k} \mathcal{Z}^{-k M} \mathbf{r}_{k M-i}(n)\right] \\
& =\sum_{i=0}^{M-1} \mathcal{Z}^{i} \mathbf{R}_{i}\left(\mathcal{Z}^{M}, n\right)
\end{aligned}
$$

where $\mathbf{R}_{i}(\mathcal{Z}, n)$ is called the $i$ th polyphase component of the type 2 representation. Note that for convenience we have used the advance operators $\mathcal{Z}^{i}$ to define type 2 polyphase representation. In the LTI case, the definitions in (3.1) and (3.2) reduce to the conventional definitions of polyphase representations [12].

Similarly, we can define the type 2 and type 1 polyphase representations for the direct form A and B implementations, respectively. However, as we explained in Section II-A, we will only use direct form $\mathbf{A}$ for decimation filters (for which type 1 polyphase is useful) and direct form $B$ for interpolation filters (for which type 2 polyphase is useful). Therefore, in the paper, we need only (3.1) and (3.2).

\section{B. Noble Identities and Efficient Structures for Decimation and Interpolation Filters}

In the LTI case, the noble identities [12] are very useful in the implementions of decimation and interpolation filters. In the LTV case, similar identities continue to hold. Consider the TV multirate systems given in Fig. 6(a) and (c), where

$$
\begin{aligned}
\mathbf{E}\left(n, \mathcal{Z}^{M}\right) & =\sum_{k} \mathbf{e}_{k}(n) \mathcal{Z}^{-k M} \\
\mathbf{R}\left(\mathcal{Z}^{M}, n\right) & =\sum_{k} \mathcal{Z}^{-k M} \mathbf{r}_{k}(n) .
\end{aligned}
$$

The noble identities say that we can redraw Fig. 6(a) as in Fig. 6(c) and Fig. 6(b) as in Fig. 6(d), where

$$
\begin{aligned}
& \mathbf{E}(M n, \mathcal{Z})=\sum_{k} \mathbf{e}_{k}(M n) \mathcal{Z}^{-k} \\
& \mathbf{R}(\mathcal{Z}, M n)=\sum_{k} \mathcal{Z}^{-k} \mathbf{r}_{k}(M n) .
\end{aligned}
$$

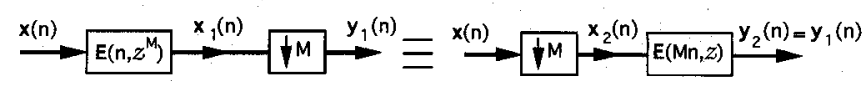

(a)

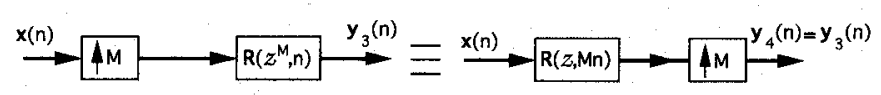

(b) (c)

(d)
Fig. 6. Time-varying noble identities for decimators and interpolators.

Notice that even though the coefficients $\mathbf{e}_{k}(n)$ and $\mathbf{r}_{k}(n)$ in (3.3) are defined for all $n$, only those coefficients at time $n=$ multiple of $M$ are relevant to the outputs (due to the decimator and interpolator). This is consistent with the fact that in the case of decimation and interpolation filters, only one out of $M$ sets of coefficients are needed (as explained in Section II-A). In the following, we will prove the noble identity for the decimator only, the proof for the interpolator being similar. From Fig. 6, we have

$$
\begin{aligned}
\mathbf{y}_{1}(n) & =\left[\mathbf{x}_{1}(m)\right]_{m=M n} \\
& =\left[\sum_{k} \mathbf{e}_{k}(m) \mathbf{x}(m-M k)\right]_{m=M n} \\
& =\sum_{k} \mathbf{e}_{k}(M n) \mathbf{x}(M n-M k) \\
\mathbf{y}_{2}(n) & =\sum_{k} \mathbf{e}_{k}(M n) \mathbf{x}_{2}(n-k) \\
& =\sum_{k} \mathbf{e}_{k}(M n) \mathbf{x}(M n-M k)=\mathbf{y}_{1}(n) .
\end{aligned}
$$

Comparing (3.5) and (3.6), we have proved the noble identity for decimator.

By using the noble identities, we find another interpretation for the polyphase components of a LTV filter. Consider the cascade system shown in Fig. 7(a), where $\mathbf{E}(n, \mathcal{Z}) \mathcal{Z}^{i}$ is sandwiched between an interpolator and a decimator. By using the type 1 polyphase representation in (3.1), Fig. 7(a) can be redrawn as Fig. $7(\mathrm{~b})$, where $\mathbf{E}_{i}\left(n, \mathcal{Z}^{M}\right)$ are as defined in (3.1). Invoking the noble identity for decimators, Fig. 7(b) can be redrawn as Fig. 7(c). Clearly, Fig. 7(c) is equivalent to Fig. 7(d). Therefore, the circuit in Fig. 7(a) is equivalent to the $i$ th polyphase component $\mathbf{E}_{i}(M n, \mathcal{Z})$ of the filter $\mathbf{E}(n, \mathcal{Z})$ and it is denoted by the following notation:

$$
\left[\mathbf{E}(n, \mathcal{Z}) \mathcal{Z}^{i}\right]_{\downarrow M}=\mathbf{E}_{i}(M n, \mathcal{Z}) .
$$

Similarly, if a direct form B filter $\mathcal{Z}^{-i} \mathbf{R}(\mathcal{Z}, n)$ is sandwiched between an interpolator and a decimator, one can show that the equivalent system is $\mathbf{R}_{i}(\mathcal{Z}, M n)$. Using the notation introduced in (3.7), we get

$$
\left[\mathcal{Z}^{-i} \mathbf{R}(\mathcal{Z}, n)\right]_{\downarrow M}=\mathbf{R}_{i}(\mathcal{Z}, M n) .
$$

1) Efficient Structures for Decimation and Interpolation Filters: Consider the decimation filter shown in Fig. 8(a), where the direct form $\mathrm{A}$ implementation of $\mathbf{E}(n, \mathcal{Z})$ is given in Fig. 1. By using the type 1 polyphase representation in (3.1) and invoking the noble identity, Fig. 8(a) can be redrawn as in Fig. 8(b), $\mathbf{E}_{i}(M n, \mathcal{Z})$ is the $i$ th type 1 polyphase component of $\mathbf{E}(m, \mathcal{Z})$ at time $m=M n$.. Similarly, the direct form 


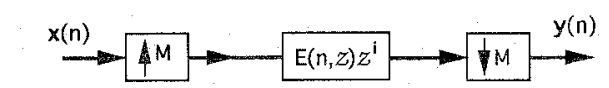

(a)

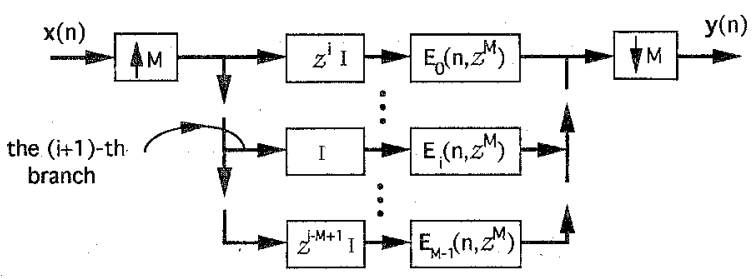

(b)

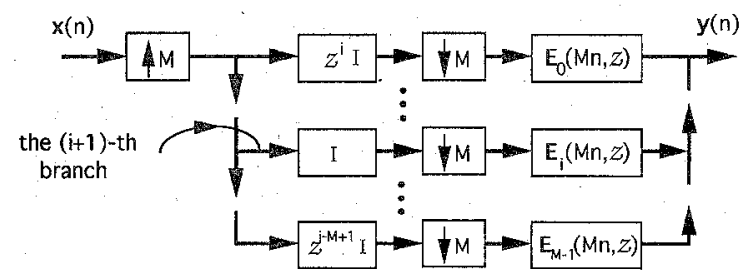

(c)

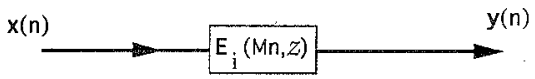

(d)

Fig. 7. Another interpretation of the $i$ th polyphase component of the filter $E(n, z)$.

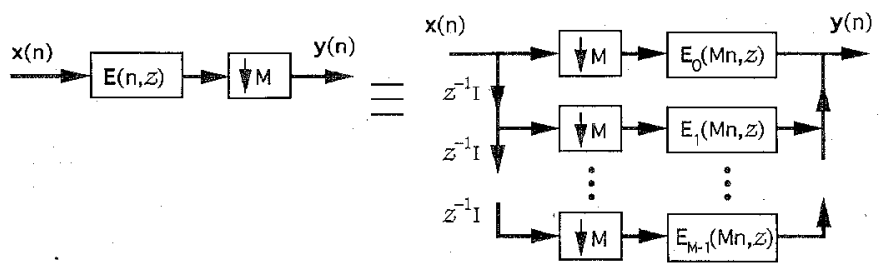

(a)

(b)

Fig. 8. Decimation filter and its efficient implementation using polyphase representation.

B implementation of the interpolation filter in Fig. 9(a) can be implemented as in Fig. $9(\mathrm{~b})$, where $\mathbf{R}_{i}(\mathcal{Z}, M n)$ is the $i$ th type 2 polyphase components of $\mathbf{R}(\mathcal{Z}, m)$ at time $m=M n$. Figs. 8(b) and 9(b) can be regarded as efficient implementations of the decimation and interpolation filters, respectively. Consider the case of fractional decimation discussed in [12, sec. 4.3.3]. If the filter is time varying, one can show that we can use both type 1 and 2 polyphase representations to simplify the circuit and arrive at an efficient structure similar to [12, Fig. 4.3-8].

\section{Block Implementation of Scalar LTV Filters}

Block implementation of LTI filters has been considered in the past [15]. In this section, we will first study the blocking

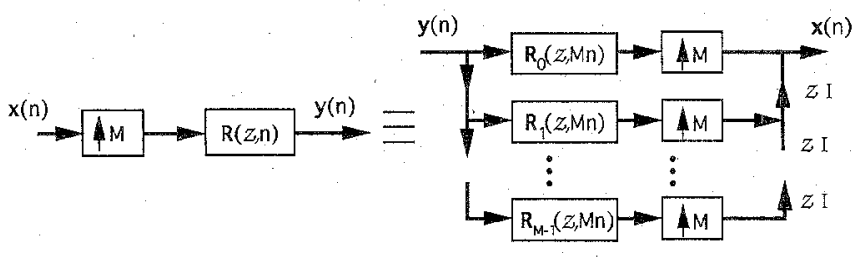

(a)

(b)

Fig. 9. Interpolation filter and its efficient implementation using polyphase representation.

and unblocking of LTV filters by using the transform domain representations. Consider the scalar LTV filter $H(n, \mathcal{Z})$. At time $n$, its type 1 polyphase components $E_{i}(n, \mathcal{Z})$ are defined in (3.1). Note that in this case, these $E_{i}(n, \mathcal{Z})$ are scalar systems. To obtain a block implementation of this scalar filter, we cascade a trivial PR FB (which contains only a delay chain and an advance chain) after the filter as shown in Fig. 10(a). Using the rule of interchanging the delay $\mathcal{Z}^{-1}$ and LTV filters given in Section II-B, we have $\mathcal{Z}^{-i} H(n, \mathcal{Z})=$ $H(n-i, \mathcal{Z}) \mathcal{Z}^{-i}$, which yields the equivalent structure in Fig. 10(b). Using the Type 1 polyphase representation for each of the filters $H(n-i, \mathcal{Z}) \mathcal{Z}^{-i}$ and applying the noble identity in Fig. 6, the block implementation can be drawn as Fig. 10(c), where the polyphase matrix $\mathbf{E}(n, \mathcal{Z})$ is shown in (3.9), at the bottom of this page, where the scalar system $E_{i}(j, \mathcal{Z})$ is the $i$ th polyphase component of $H(j, \mathcal{Z})$. From (3.9), the following. statements can be verified by directly evaluating the impulse response $h_{k}(n)$.

1) The scalar system $H(n, \mathcal{Z})$ is FIR if and only if the MIMO system $\mathbf{E}(M n, \mathcal{Z})$ is FIR.

2) If the polyphase matrix $\mathbf{E}(M n, \mathcal{Z})$ does not depend on $n$, then the scalar filter $H(n, \mathcal{Z})$ is a linear periodically time-varying filter of period $M$. This implies the filter coefficients satisfy $h_{k}(M l+n)=h_{k}(n)$.

3) If the polyphase components satisfy $E_{i}(M n-j, \mathcal{Z})=$ $E_{i}(M n, \mathcal{Z})$ or $E_{i}(M n+j, \mathcal{Z})=E_{i}(M n, \mathcal{Z})$ for $0 \leq j \leq M-1$, the polyphase matrix is said to be time-varying pseudocirculant $(M)$. In this case, the filter coefficients satisfy $h_{k}(M l-i)=h_{k}(M l)$ or $h_{k}(M l+i)=h_{k}(M l)$ for $0 \leq i \leq M-1$.

4) $E_{i}(M n-j, \mathcal{Z})$ are independent of both $j$ and $n$ (which implies both 2 and 3 ), if and only if the polyphase matrix $\mathbf{E}(n, \mathcal{Z})$ is time-invariant pseudocirculant. Therefore, the scalar filter $H(n, \mathcal{Z})$ is LTI, if and only if, both 2 and 3 are true.

Referring to Fig. 10(c), it is clear that $\sum_{n}|x(n)|^{2}=$ $\sum_{n} \mathrm{x}^{\dagger}(n) \mathrm{x}(n)$ and $\sum_{n}|y(n)|^{2}=\sum_{n} \mathbf{y}^{\dagger}(n) \mathrm{y}(n)$, since the delay chain and advance chain are simply the mechanisms of blocking and unblocking. Therefore, scalar system from $x(n)$ to $y(n)$ (i.e., the system $H(n, \mathcal{Z})$ in Fig. $10(a)$ ) is lossless

$$
\mathbf{E}(n, \mathcal{Z})=\left[\begin{array}{cccc}
E_{0}(M n, \mathcal{Z}) & E_{1}(M n, \mathcal{Z}) & \cdots & E_{M-1}(M n, \mathcal{Z}) \\
E_{M-1}(M n-1, \mathcal{Z}) \mathcal{Z}^{-1} & E_{0}(M n-1, \mathcal{Z}) & \cdots & E_{M-2}(M n-1, \mathcal{Z}) \\
\vdots & \vdots & \ddots & \vdots \\
E_{1}(M n-M+1, \mathcal{Z}) \mathcal{Z}^{-1} & E_{2}(M n-M+1, \mathcal{Z}) \mathcal{Z}^{-1} & \cdots & E_{0}(M n-M+1, \mathcal{Z})
\end{array}\right]
$$




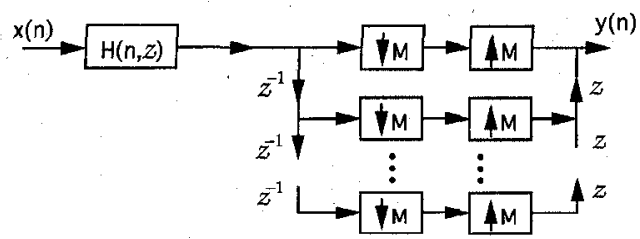

(a)

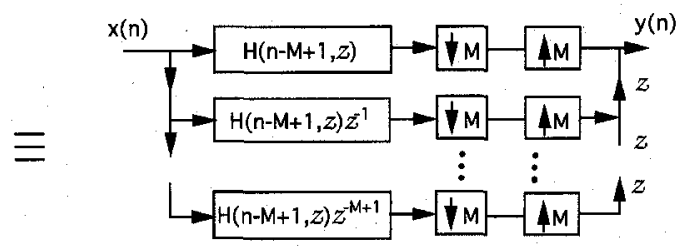

(b)

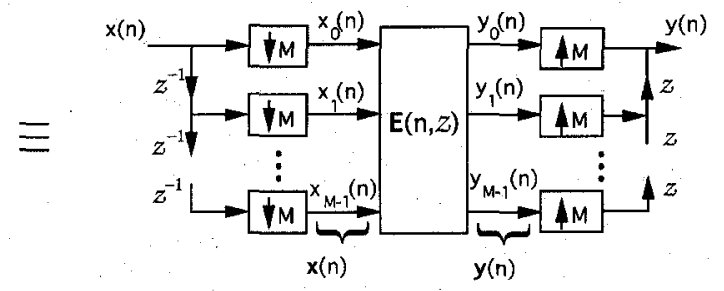

(c)

Fig. 10. Block implementation of the scalar LTV filter $H(n, \mathcal{Z})$, where the matrix $\mathbf{E}(n, \mathcal{Z})$ is defined in (3.9).

(passive) if and only if the MIMO system $\mathbf{E}(M n, \mathcal{Z})$ in Fig. 10(c) is lossless (correspondingly passive). In particular, all MIMO LTI paraunitary (PU) systems are lossless. If the MIMO system $\mathbf{E}(M n, \mathcal{Z})$ in (3.9) is chosen as a general LTI PU system, then the resulting scalar system $H(n, \mathcal{Z})$ obtained by unblocking mechanism is lossless. Thus we conclude that there are nontrivial scalar FIR LTV lossless systems. The invertibility of the scalar systems obtained by unblocking is clearly equivalent to the invertibility of the original MIMO system. Let $\mathbf{R}(\mathcal{Z}, M n)$ be the blocked version of another filter $F(\mathcal{Z}, n)$. It is clear that $\mathbf{R}(\mathcal{Z}, M n)$ is the inverse of $\mathbf{E}(M n, \mathcal{Z})$ if and only if $F(\mathcal{Z}, n)$ is the inverse of $H(n, \mathcal{Z})$. The idea of block implementation is useful, since it can generate some examples to illuminate the theory of TVFB, such as the existence of nontrivial scalar FIR lossless system in Example 6.1 and the following $2 \times 2$ system, which is shown to be unfactorizable in Section IV of a companion paper [10].

Consider an $M \times M$ paraunitary LTI system $\mathbf{E}(z)$. Let $M_{1}$ be a factor $M$. Then we can obtain a $M_{1} \times M_{1}$ lossless LTV system by partially unblocking $\mathbf{E}(z)$. To show how this can be done, we provide a simple example in the following.

Example 3.1-MIMO Lossless LTV Systems from Unblocking: Let $\mathbf{E}(z)$ be chosen as the following scaled $4 \times 4$ (permuted) Hadamard matrix:

$$
\mathbf{T}=\frac{1}{2}\left[\begin{array}{rrrr}
1 & 1 & 1 & 1 \\
1 & 1 & -1 & -1 \\
1 & -1 & -1 & 1 \\
1 & -1 & 1 & -1
\end{array}\right]
$$

Clearly, $\mathbf{T}$ is unitary. In order to obtain a causal system, we use a delay chain at the synthesis end instead of an

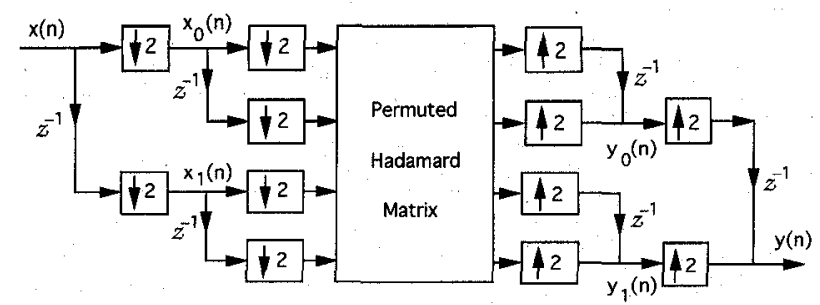

Fig. 11. Example 3.1-A MIMO lossless system obtained by partial unblocking.

advance chain. After some rearrangements, we can get Fig. 11 . Defining $\mathbf{x}(n)=\left[x_{0}(n) x_{1}(n)\right]^{T}$ and $\mathbf{y}(n)=\left[y_{0}(n) y_{1}(n)\right]^{T}$, we obtain the following $2 \times 2$ system $\mathcal{H}: \mathbf{y}(n)=\mathbf{e}_{0}(n) \mathbf{x}(n)+$ $\mathbf{e}_{1}(n) \mathbf{x}(n-1)+\mathbf{e}_{2}(n) \mathbf{x}(n-2)$, where the coefficients are given by

$$
\begin{aligned}
\mathbf{e}_{0}(2 n) & =\frac{1}{2}\left[\begin{array}{rr}
1 & -1 \\
1 & 1
\end{array}\right] \\
\mathbf{e}_{1}(2 n) & =\frac{1}{2}\left[\begin{array}{rr}
-1 & 1 \\
1 & -1
\end{array}\right] \\
\mathbf{e}_{2}(2 n) & =0 \\
\mathbf{e}_{0}(2 n+1) & =0 \\
\mathbf{e}_{1}(2 n+1) & =\frac{1}{2}\left[\begin{array}{rr}
1 & 1 \\
1 & -1
\end{array}\right], \\
\mathbf{e}_{2}(2 n+1) & =\frac{1}{2}\left[\begin{array}{rr}
1 & 1 \\
-1 & -1
\end{array}\right]
\end{aligned}
$$

The above $2 \times 2$ system $\mathcal{H}$ is lossless since $\mathbf{T}$ is unitary. Its inverse which can be obtained by unblocking $\mathbf{T}^{\dagger}$, is also FIR. Thus, $\mathcal{H}$ is a FIR lossless system with FIR inverse.

\section{POLYPHASE APPROACH TO TIME-VARYING FILTER BANKS AND TRANSMULTIPLEXERS}

Consider the TVFB given in Fig. 12(a). As we explained in Section II-A, for convenience we will choose direct form A for the analysis filters $H^{k}(n, \mathcal{Z})$ and direct form $\mathrm{B}$ for the synthesis filters $F^{k}(\mathcal{Z}, n)$. Using the transform domain descriptions, the filters can be expressed as follows:

$$
H^{k}(n, \mathcal{Z})=\sum_{i} h_{i}^{k}(n) \mathcal{Z}^{-i}
$$

and

$$
F^{k}(\mathcal{Z}, n)=\sum_{i} \mathcal{Z}^{-i} f_{i}^{k}(n), \quad \forall n
$$

where the superscript $k$ is used to denote the filter number. The subband signals $y_{k}(n)$ and the output signal $\hat{x}(n)$ (as shown in Fig. 12(a) can, respectively, be expressed as

$$
\begin{aligned}
& y_{k}(n)=\sum_{l} h_{l}^{k}(M n) x(M n-l) \\
& \hat{x}(n)=\sum_{k=0}^{M-1} \sum_{m} y_{k}(m) f_{n-M m}^{k}(M m) .
\end{aligned}
$$

From the above equation, it is clear that both the output and decimated subband signals are independent of those filter coefficients $h_{i}^{k}(n)$ that occur at $n \neq$ integer multiple of $M$. Therefore, we need to consider only the coefficients 


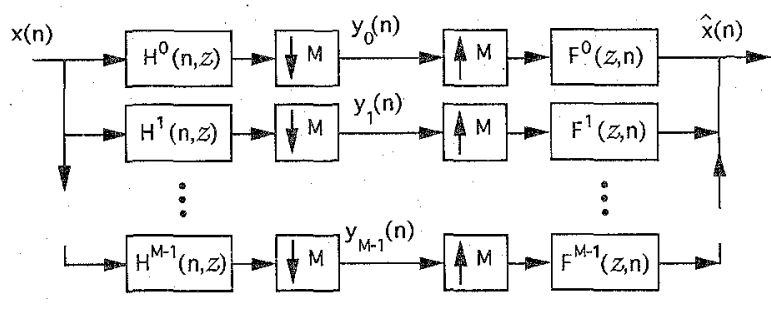

(a)

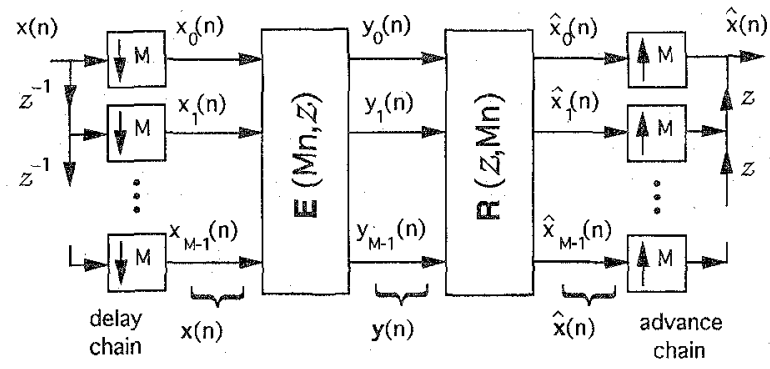

(b)

Fig. 12. Time-varying filter bank and its polyphase implementation.

$h_{i}^{k}(M n)$ and $f_{i}^{k}(M n)$. Applying the proposed polyphase representations, Fig. 12(a) can be redrawn as Fig. 12(b), where the noble identities have been invoked to move the polyphase matrices. The polyphase matrices $\mathbf{E}(M n, \mathcal{Z})$ and $\mathbf{R}(\mathcal{Z}, M n)$ are, respectively, defined as follows:

$$
\begin{aligned}
& E_{k j}(M n, \mathcal{Z})=\left[H^{k}(n, \mathcal{Z}) \mathcal{Z}^{j}\right]_{\downarrow M} \\
& R_{j k}(\mathcal{Z}, M n)=\left[\mathcal{Z}^{-j} F^{k}(\mathcal{Z}, n)\right]_{\downarrow M}
\end{aligned}
$$

where the notation []$_{\downarrow M}$ is defined in (3.7) and (3.8). In other words, the $k j$ th element of $\mathbf{E}(M n, \mathcal{Z})$ and $j k$ th element of $\mathbf{R}(\mathcal{Z}, M n)$ are, respectively, the $j$ th polyphase component of $H^{k}(n, \mathcal{Z})$ and $F^{k}(\mathcal{Z}, n)$. The relation between the analysis/synthesis filters and the polyphase matrices can be described as

$$
\begin{aligned}
& {\left[H^{0}(M n, \mathcal{Z}) \cdots H^{M-1}(M n, \mathcal{Z})\right]^{T}} \\
& =\mathbb{E}\left(M n, \mathcal{Z}^{M}\right)\left[\begin{array}{lll}
1 & \mathcal{Z}^{-1} & \ldots \mathcal{Z}^{-M+1}
\end{array}\right]^{T} \\
& {\left[F^{0}(\mathcal{Z}, M n) \cdots F^{M-1}(\mathcal{Z}, M n)\right]} \\
& =\left[\begin{array}{llll}
1 & \mathcal{Z} & \cdots & \mathcal{Z}^{M-1}
\end{array}\right] \mathbf{R}\left(\mathcal{Z}^{M}, M n\right) .
\end{aligned}
$$

Only $H^{k}(M n, \mathcal{Z})$ and $F^{k}(\mathcal{Z}, M n)$ are related to the polyphase matrices. $H^{k}(M n-i, \mathcal{Z})$ and $F^{k}(\mathcal{Z}, M n-i)$ are irrelevant to the output of the $\mathrm{FB}$, for any $i \neq$ multiple of $M$. Because of the polyphase representation, we can characterize all TVFB's by characterizing the MIMO systems $\mathrm{E}(M n, \mathcal{Z})$ and $\mathbf{R}(\mathcal{Z}, M n)$.

\section{A. Time-Varying Perfect Reconstruction Filter Bank}

From the polyphase implementation of the TV FB shown in Fig. $12(\mathrm{~b})$, it is clear that the FB achieves perfect reconstruction (PR) (i.e., $\hat{x}(n)=x(n)$ ), if and only if

$$
\mathbf{R}(\mathcal{Z}, M n) \mathbb{E}(M n, \mathcal{Z})=\mathbb{I}, \quad \forall n .
$$

In other words, we obtain $\mathrm{PR}$ if $\mathbf{R}(\mathcal{Z}, M n)$ is the inverse of the MIMO filter $\mathbb{E}(M n, \mathcal{Z})$. In the general LTV case, (4.5) does not imply $\mathbb{E}(M n, \mathcal{Z}) \mathbf{R}(\mathcal{Z}, M n)=\mathbf{I}$. To see this, consider Example 1.1, where an one-channel TVFB is given. In this example, $M=1$, so $\mathrm{E}(M n, \mathcal{Z})$ and $\mathbf{R}(\mathcal{Z}, M n)$ are, respectively, the scalar filters $\mathcal{H}$ and $\mathcal{G}$. Clearly, $\mathbf{E}(M n, \mathcal{Z}) \mathbf{R}(\mathcal{Z}, M n) \neq \mathbf{I}$ because $\mathbf{R}(\mathcal{Z}, M n)$ (in this case, $\mathcal{G}$ ) is not invertible! Therefore, in general, if we interchange the analysis and synthesis polyphase matrix of a PR TVFB, the PR property will be destroyed. This is very different from the conventional PR LTI FB.

However, if the matrix $\mathbf{R}(\mathcal{Z}, M n)$ is also invertible, then its inverse is unique and it is $\mathbb{E}(M n, \mathcal{Z})$. To prove this, let $\mathbb{R}^{-1}(\mathcal{Z}, M n)$ be any inverse of $\mathbb{R}(\mathcal{Z}, M n)$. Premultiplying both sides of (4.5) by $\mathbf{R}^{-1}(\mathcal{Z}, M n)$, we get $\mathbf{R}^{-1}(\mathcal{Z}, M n)=\mathbf{E}(M n, \mathcal{Z})$. In this case, the cascade $\mathbf{E}(M n, \mathcal{Z}) \mathbf{R}(\mathcal{Z}, M n)=\mathbf{I}$. This implies the following relation between the analysis and synthesis filters:

$$
\begin{aligned}
& {\left[H^{k}(n, \mathcal{Z}) F^{l}(\mathcal{Z}, n)\right]_{\downarrow M}} \\
& =\sum_{i=0}^{M-1} E_{k i}(M n, \mathcal{Z}) R_{i l}(\mathcal{Z}, M n)=\delta(k-l), \forall n .
\end{aligned}
$$

The above equation can be viewed as a generalization of the biorthogonality condition of the analysis/synthesis filters defined for the LTI case [13], [19]. To illustrate the above theory, we provide an example in the following.

1) Example 4.1-PR FIR TVFB: Consider the $M$ channel TVFB whose polyphase matrices are

$$
\begin{aligned}
\mathbf{E}(M n, \mathcal{Z})= & {\left[\mathbb{I}-\mathbf{u}(n) \mathbf{v}^{\dagger}(n)\right] } \\
& +\mathbf{u}(n) \mathbf{v}^{\dagger}(n-1) \mathcal{Z}^{-1} \\
\mathbf{R}(\mathcal{Z}, M n)= & {\left[\mathbf{I}+\mathbf{u}(n) \mathbf{v}^{\dagger}(n)\right] } \\
& -\mathcal{Z}^{-1} \mathbf{u}(n+1) \mathbf{v}^{\dagger}(n)
\end{aligned}
$$

where the vectors satisfy $\mathbf{v}^{\dagger}(n) \mathbf{u}(n)=0$ for all $n$. One can verify by direct substitution that the polyphase matrices in (4.7) satisfy $\mathbf{R}(\mathcal{Z}, M n) \mathbf{E}(M n, \mathcal{Z})=\mathbf{E}(M n, \mathcal{Z}) \mathbf{R}(\mathcal{Z}, M n)=\mathbf{I}$. Therefore, the TVFB achieves PR and the synthesis polyphase matrix is invertible. The analysis and synthesis filters are, respectively

$$
\begin{aligned}
H^{k}(M n, \mathcal{Z})= & {\left[1-u_{k}(n) v_{k}(n)\right] \mathcal{Z}^{-k} } \\
& -\sum_{i \neq k} u_{k}(n) v_{i}(n) \mathcal{Z}^{-i} \\
& +\sum_{i=0}^{M-1} u_{k}(n) v_{i}(n-1) \mathcal{Z}^{-M-i} \\
F^{k}(\mathcal{Z} ; M n)= & \mathcal{Z}^{k}\left[1+u_{k}(n) v_{k}(n)\right] \\
& +\sum_{i \neq k} \mathcal{Z}^{i} u_{i}(n) v_{k}(n) \\
& -\sum_{i=0}^{M-1} \mathcal{Z}^{-M+i} u_{i}(n) v_{k}(n-1)
\end{aligned}
$$

where $u_{i}(n)$ and $v_{i}(n)$ are, respectively, the $i$ th element of $\mathbf{u}(n)$ and $\mathbf{v}(n)$. We have a PR TVFB where both its analysis and synthesis filters are causal FIR. One can verify that the filters satisfy the biorthogonal condition in (4.6). 
Remark: Applying the result in Section III-C, we know that the TVFB reduces to a scalar LTI system if and only if the cascade $\mathbf{R}(\mathcal{Z}, n) \mathbf{E}(n, \mathcal{Z})$ is a time-invariant pseudocirculant matrix. Compare this with the result that an LTI FB reduces to a scalar LTI system if and only if it is alias free [12].

2) The Tilde Operation: In the LTI case, the tilde operation was used in [12] to the study of conventional FB's. Given an LTI system $\mathbf{E}(z)$, the tilde operation is defined as $\tilde{\mathbf{E}}(z)=\mathbf{E}^{\dagger}\left(1 / z^{*}\right)$. This tilde operation was proved to be very useful in the analysis of conventional $\mathrm{FB}$, especially for the LTI PU FB's. In the LTV case, we will define a similar operation. The time-varying tilde operation consists of the three following steps.

1) Replace the multipliers $\mathbf{e}_{k}(n)$ and $\mathbf{r}_{k}(n)$ with their transpose complex-conjugates, $\mathbf{e}_{k}^{\dagger}(n)$, and $\mathbf{r}_{k}^{\dagger}(n)$, respectively.

2) Interchange the multiplier and the delay, i.e., $\mathbf{e}_{k}(n) \mathcal{Z}^{-k}$ is replaced with $\mathcal{Z}^{-k} \mathbf{e}_{k}(n)$ and $\mathcal{Z}^{-k} \mathbf{r}_{k}(n)$ is replaced with $\mathbf{r}_{k}(n) \mathcal{Z}^{-k}$. Note that this operation will change the direct form A to the direct form B structure, and vice versa.

3) Replace the delay $\mathcal{Z}^{-1}$ with the advance element $\mathcal{Z}$.

By using the above definition, the tilde operation on LTV filters can be described as

$$
\begin{aligned}
& \tilde{\mathbf{E}}(n, \mathcal{Z})=\sum_{k} \mathcal{Z}^{k} \mathrm{e}^{\dagger}(n) \\
& \tilde{\mathbf{R}}(\mathcal{Z}, n)=\sum_{k} \mathbf{r}_{k}^{\dagger}(n) \mathcal{Z}^{k}
\end{aligned}
$$

Note that $\mathbf{E}(n, \mathcal{Z})$ and $\mathbf{R}(\mathcal{Z}, n)$ are, respectively, in direct form $\mathrm{A}$ and $\mathrm{B}$ while $\tilde{\mathbf{E}}(n, \mathcal{Z})$ and $\tilde{\mathbf{R}}(\mathcal{Z}, n)$ are, respectively, in direct form $B$ and $A$. The tilde operation will be shown to be very useful as we shall repeatedly see later. Note that the tilde operation is its own inverse, i.e., $\tilde{\tilde{\mathbf{E}}}(n, \mathcal{Z})=\mathbf{E}(n, \mathcal{Z})$. Moreover, the tilde of a cascade of two LTV filters satisfies the following property:

Lemma 4.1: Consider two LTV systems $\mathcal{G}_{0}$ followed by $\mathcal{G}_{1}$. The tilde of the cascade is $\widetilde{\mathcal{G}_{1} \mathcal{G}_{0}}=\tilde{\mathcal{G}}_{0} \tilde{\mathcal{G}}_{1}$.

Proof: We will only prove the fact for the case when both $\mathcal{G}_{0}$ and $\mathcal{G}_{1}$ are in direct form A implementation, namely $\mathbf{E}^{0}(n, \mathcal{Z})$ and $\mathbf{E}^{1}(n, \mathcal{Z})$, respectively. The proof for other cases is similar. Using (2.8) and (4.9), we get

$$
\begin{aligned}
\text { tilde } & {\left[\mathbf{E}^{1}(n, \mathcal{Z}) \mathbf{E}^{0}(n, \mathcal{Z})\right] } \\
& =\text { tilde }\left[\sum_{k, l} \mathbf{e}_{k}^{1}(n) \mathbf{e}_{l}^{0}(n-k) \mathcal{Z}^{-k-l}\right] \\
& =\sum_{k, l} \mathcal{Z}^{k+l} \mathbf{e}_{l}^{0 \dagger}(n-k) \mathbf{e}_{k}^{1 \dagger}(n) \\
& =\sum_{k, l} \mathcal{Z}^{l} \mathbf{e}_{l}^{0 \dagger}(n) \mathcal{Z}^{k} \mathbf{e}_{k}^{1 \dagger}(n) \\
& =\tilde{\mathbf{E}}^{0}(n, \mathcal{Z}) \tilde{\mathbf{E}}^{1}(n, \mathcal{Z})
\end{aligned}
$$

The proof is complete.

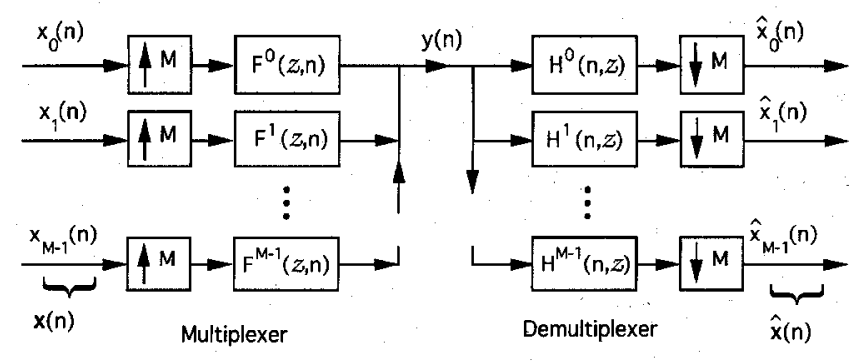

Fig. 13. Time-varying transmultiplexer.

3) Interchangability of the Analysis and Synthesis of a PR TVFB: In the LTI case, if $\left\{H^{k}(z), F^{k}(z)\right\}$ forms a conventional PR $\mathrm{FB},\left\{F^{k}(z), H^{k}(z)\right\}$ will also form a PR analysis/synthesis system of [12, Problem 5.17]. In the LTV case, if we directly interchange $H^{k}(n, \mathcal{Z})$ with $F^{k}(\mathcal{Z}, n)$ (and vice versa) without any modification, the PR property in general does not continue to hold. To see this, consider Example 1.1, where we show a one channel PR TVFB with $\mathcal{H}$ as the only analysis filter and $\mathcal{G}$ as the only synthesis filter. Clearly, interchanging $\mathcal{G}$ with $\mathcal{H}$ does not preserve the PR property because $\mathcal{G}$ is not invertible! The proper way of interchanging the analysis and synthesis filters is described in the following theorem, which follows directly from (4.4) and Lemma 4.1.

4) Theorem 4.1-Interchanging the Analysis and Synthesis Filters: Let $\left\{H^{k}(n, \mathcal{Z}), F^{k}(\mathcal{Z}, n)\right\}$ be respectively, the analysis and synthesis filters of a PR TVFB, then the FB with $\tilde{F}^{k}(\mathcal{Z}, n)$ as the analysis filters and $\tilde{H}^{k}(n, \mathcal{Z})$ as the synthesis filters also achieves PR.

\section{B. Time-Varying Perfect Reconstruction Transmultiplexers}

Transmultiplexers have been used in communication to convert between two formats called time-division multiplexed (TDM) format and frequency-division multiplexed (FDM) format. The application of conventional FB theory to transmultiplexers was studied in [12] and [16]. In this subsection, we will generalize the results in [12] and [16] to the more general LTV case. Consider Fig. 13, where a TV transmultiplexer is shown. In the traditional theory of transmultiplexers, we have the following two special cases: i) When $F^{k}(\mathcal{Z}, n)=\mathcal{Z}^{k}$ and $H^{k}(n, \mathcal{Z})=\mathcal{Z}^{-k}, y(n)$ is a TDM signal; ii) When $F^{k}(\mathcal{Z}, n)$ and $H^{k}(n, \mathcal{Z})$ are LTI ideal bandpass filters, $y(n)$ is a FDM signal. In the above two cases, it is clear that $\hat{\mathbf{x}}(n)=\mathbf{x}(n)$, i.e., the transmultiplexer achieves PR. By using the theory for PR LTI FB's, the author in [16] showed that PR LTI transmultiplexer is possible by using nonideal LTI filters. More precisely, it was shown that if the LTI filters $\left\{H^{k}(z), F^{k}(z)\right\}$ form a PR analysis/synthesis system, then the corresponding LTI transmultiplexer achieves PR. In the more general LTV case, a similar statement does not hold as shown in the following: By using the polyphase representation, Fig. 13 can be redrawn as Fig. 14, where the elements of the matrices $\mathbf{E}(M n, \mathcal{Z})$ and $\mathbf{R}(\mathcal{Z}, M n)$ are defined in (4.3). It is clear from Fig. 14 that the TV transmultiplexer achieves PR if and only if $\mathbf{E}(M n, \mathcal{Z}) \mathbf{R}(\mathcal{Z}, M n)=$ I. However, from the previous subsection, we know that the PR property of $\left\{H^{k}(n, \mathcal{Z}), F^{k}(\mathcal{Z}, n)\right\}$ does not imply 


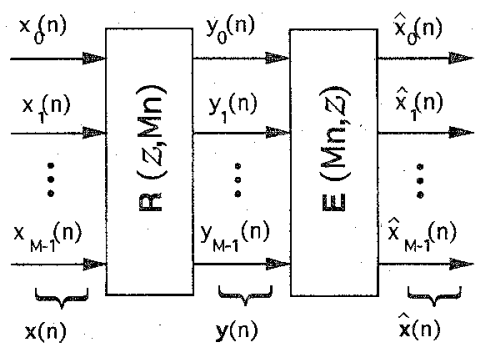

Fig. 14. Redrawing of the time-varying transmultiplexer by using the polyphase representation.

$\mathbb{E}(M n, \mathcal{Z}) \mathbf{R}(\mathcal{Z}, M n)=\mathbf{I}$. Therefore, the transmultiplexer in Fig. 13 may not be PR even if $\left\{H^{k}(n, \mathcal{Z}), F^{k}(\mathcal{Z}, n)\right\}$ forms a PR TVFB unless the synthesis system is also invertible (which is not always true). In order to achieve PR TV transmultiplexer, we can use the result of Lemma 4.1 to show that PR is attained if $\tilde{H}^{k}(n, \mathcal{Z})$ are used as the filters in the multiplexer and $\tilde{F}^{k}(\mathcal{Z}, n)$ are used as the filters in the demultiplexer.

\section{Lossless Time-VArying Filters and. Filter Banks}

In the previous section, using the polyphase representation, we have shown that we can characterize all TVFB's by characterizing MIMO LTV filters. Moreover, the analysis bank preserves the energy from the input to the subband signals $y_{k}(m)$ (as shown in Fig. 12) if and only if the corresponding analysis polyphase matrix is lossless. In this section, we will first give both the impulse response and transform domain characterizations of lossless filters and their inverses. Then some subtle properties of the inverses of lossless filters are studied in detail. We will also derive the properties of lossless TVFB's. Lossless TVFB's are important in the applications of subband coding [12].

\section{A. Characterizations of Lossless LTV}

Filters and Their Inverses

1) Impulse Response Characterization: Consider the following MIMO LTV system:

$$
\mathbf{y}(n)=\sum_{k} \mathbf{e}_{k}(n) \mathbf{x}(n-k) .
$$

From the definition given in Section I-B, the above system is lossless if $\sum_{n} \mathbf{y}^{\dagger}(n) \mathbf{y}(n)=\sum_{n} \mathbf{x}^{\dagger}(n) \mathbf{x}(n)$ for any $\mathbf{x}(n) \epsilon$ $l_{2}(M)$. Using (5.1), we have

$$
\sum_{n} \mathbf{y}^{\dagger}(n) \mathbf{y}(n)=\sum_{k, l, n} \mathbf{x}^{\dagger}(n-k) \mathbf{e}_{k}^{\dagger}(n) \mathbf{e}_{l}(n) \mathbf{x}(n-l) .
$$

Making a change of variable, we get the following:

$$
\begin{aligned}
& \sum_{n} \mathbf{y}^{\dagger}(n) \mathbf{y}(n) \\
& =\sum_{i, j} \mathbf{x}^{\dagger}(i) \underbrace{\left[\sum_{k} \mathbf{e}_{k}^{\dagger}(k+n) \mathbf{e}_{k+i-j}(k+n)\right]}_{\mathbf{T}_{i j}} \mathbf{x}(j) .
\end{aligned}
$$

Since the input $\mathbf{x}(n)$ is arbitrary, the right-hand side of (5.3) equals $\sum_{n} \mathbf{x}^{\dagger}(n) \mathbf{x}(n)$ if and only if the matrices $\mathbf{T}_{i j}$ satisfy
$\mathbf{T}_{i j}=\mathbf{I} \delta(i-j)$. Therefore, we conclude that the system in (5.1) is lossless if and only if

$$
\sum_{k} \mathbf{e}_{k}^{\dagger}(k+n) \mathbf{e}_{k+l}(k+n)=\mathbb{I} \delta(l), \quad \forall n .
$$

Note that the left-hand side of (5.4)' is different from $\sum_{k} \mathbf{e}_{k}^{\dagger}(n) \mathbf{e}_{k+l}(n)$. In the LTI case, (5.4) reduces to $\sum_{k} \mathbf{e}_{k}^{\dagger} \mathbf{e}_{k+l}=\mathbb{I} \delta(l)$. This is consistent with the paraunitary condition [12] $\mathbf{E}^{\dagger}\left(1 / z^{*}\right) \mathbf{E}(z)=\mathbf{I}$, where $\mathbf{E}(z)=\sum_{k} \mathbf{e}_{k} z^{-k}$.

For the direct form B implementation of LTV filter, which can be described as $\mathbf{y}(n)=\sum_{k} \mathbf{r}_{k}(n-k) \mathbf{x}(n-k)$, the lossless condition for the direct form $\mathrm{B}$ can be obtained by simply replacing the coefficients $\mathrm{e}_{k}(n)$ in (5.4) with $\mathbf{r}_{k}(n-k)$. Therefore, a direct form B system is lossless if and only if $\sum_{k} \mathrm{r}_{k}^{\dagger}(n) \mathbf{r}_{k+l}(n-l)=\mathbf{I} \delta(l)$ for all $n$.

a) Lossless LTV FIR systems: Assume that the LTV filter described in (5.1) is an $N$ th-order system, i.e., $\mathbf{y}(n)=$ $\sum_{k=0}^{N} \mathbf{e}_{k}(n) \mathbf{x}(n-k)$. Then by substituting $l=N$ into the lossless condition in (5.4), we obtain $\mathbf{e}_{0}^{\dagger}(n) \mathbf{e}_{N}(n)=0$ for all $n$. That is, the lowest and highest order coefficients of a lossless FIR system are both singular for each $n$ (if neither of them equals to the null matrix). The sum of their rank cannot exceed $M$. In [10], we will see that this property is very useful in the factorization of lossless LTV systems in terms of smaller building blocks.

b) Nonlosslessness of the frozen system: Consider the following system: $\mathbf{y}(n)=\sum_{k} \mathrm{e}_{k}(L) \mathbf{x}(n-k)$, where $L$ is some fixed integer. The above system can be thought as the system in (5.1) with the coefficients frozen at time $L$. Note that in general the frozen system, which is an LTI system, does not satisfy the paraunitary condition. Therefore, the frozen system might not be lossless.

c) Replacing delay $z^{-1}$ with $z^{L}$ : We know that if we replace the delay $z^{-1}$ in an implementation of a lossless LTI system with $z^{L}$ for some integer $L$, the system remains lossless. This is not true in general for the LTV case! To see this, consider Example 1.1. If we replace the delay $Z^{-1}$ in the structure of Fig. 1 with an advance operator $\mathcal{Z}^{L}$, the new system will be $y(n)=h_{0}(n) x(n)+h_{1}(n) x(n+L)$ with $h_{0}(n)$ and $h_{1}(n)$ given in (1.1). The new system is no longer lossless because the samples $x(0), \cdots, x(L)$ are missing. In the following, we will show how to modify the coefficients such that losslessness is preserved under such transformation.

Theorem 5.1-Delay Transformation: Consider a lossless system $\mathrm{e}_{k}(n), 0 \leq k \leq N$, shown in Fig. 1 . If the delay $z^{-1}$ is replaced with $z^{-L}$, the direct form $\mathrm{A}$ implementations in Fig. 1 will remain lossless provided that the coefficients for the new system are obtained as

$$
\begin{aligned}
& \hat{\mathrm{e}}_{k}(L n+i)=\mathrm{e}_{k}(n) \\
& \hat{\mathrm{e}}_{k}(L n-i)=\mathrm{e}_{k}(n) \quad \text { for } 0 \leq i \leq L-1, \quad \forall n .
\end{aligned}
$$

or

Similarly, if we replace $z^{-1}$ with $z^{-L}$ in the direct form B shown in Fig. 2, then the system will remain lossless if $\hat{\mathbf{r}}_{k}(\operatorname{Ln} \pm i)=\mathbf{r}_{k}(n)$.

Theorem 5.1 can be proved by direct substitution. Recall from Section III-C that a LTV system is TV pseudocirculant 


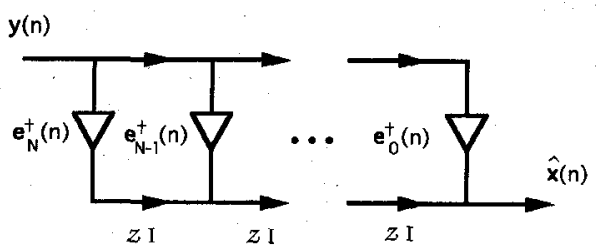

Fig. 15. Inverse of the lossless system in Fig. 1.

$(M)$ if $\mathbf{e}_{k}(M n-i)=\mathbf{e}_{k}(M n)$ or $\mathbf{e}_{k}(M n+i)=\mathbf{e}_{k}(M n)$ for $0 \leq i \leq M-1$. Therefore, a lossless system remains lossless under the delay transformation if and only if it is TV pseudocirculant $(L)$.

2) The Inverses for Lossless LTV Filters: For the general LTV filter, it is not easy to determine if the filter is invertible and find its inverse if it is invertible. However, if the LTV filter is lossless, it is always invertible. Moreover the inverse is FIR if the lossless system is. FIR. However unlike the LTI case, the inverse may not be unique, invertible, or lossless, as we have demonstrated in Example 1.1. In this subsection, we will construct an anticausal inverse for any LTV lossless system. In the next subsection, we will show that the invertibility and the losslessness of the inverse are closely related to each other.

In the LTI case, given a MIMO paraunitary system $\mathbf{E}(z)$, we know [12] that the inverse is simply $\mathbf{E}^{\dagger}\left(1 / z^{*}\right)$. This suggests that the inverse of an LTV lossless system of the form in Fig. 1 might be in the form in Fig. 15. This indeed is the case, as verified next. The output of the system in Fig. 15 can be written as

$$
\hat{\mathbf{x}}(n)=\sum_{k} \mathbf{e}_{k}^{\dagger}(n+k) \mathbf{y}(n+k) .
$$

Substituting (5.1) into the above equation and simplifying the result, we have

$$
\hat{\mathbf{x}}(n)=\sum_{l}\left[\sum_{k} \mathbf{e}_{k}^{\dagger}(n+k) \mathbf{e}_{k+l}(n+k)\right] \mathbf{x}(n-l) .
$$

Applying the lossless condition (5.4) to the above equation, we have $\hat{\mathbf{x}}(n)=\mathbf{x}(n)$ for all $n$. Therefore, we have shown the following.

Theorem 5.2: All lossless LTV systems are invertible. Moreover, the inverse is FIR as in Fig. 15 if the original lossless system is FIR as in Fig. 1.

Unlike the LTI case, we will see later that the inverse of a LTV lossless system may not be unique. Notice that the anticausal inverse given above is implemented in direct form B. Except for this, everything is similar to the LTI case, i.e., the coefficients are mirror-image and transpose-conjugates of the coefficients of the original system. By using a procedure similar to that of Section V-A, one can show that the inverse system in Fig. 15 is lossless if and only if

$$
\sum_{k} \mathbf{e}_{k}(n) \mathbf{e}_{k+l}^{\dagger}(n+l)=\mathbf{I} \delta(l) \quad \forall n .
$$

Therefore, if the coefficients of the system in Fig. 1 satisfy both (5.4) and (5.8), then it is a lossiless system with a lossless inverse. In the LTI case, (5.4) and (5.8), respectively, reduce to the conditions $\sum_{k} \mathbf{e}_{k}^{\dagger} \mathbf{e}_{k+l}=\mathbf{I} \delta(l)$ and $\sum_{k} \mathbf{e}_{k} \mathbf{e}_{k+l}^{\dagger}=\mathbf{I} \delta(l)$.
The two conditions in the LTI case are equivalent to each other. Therefore, the inverse of a LTI PU system is also PU. However, in the LTV case, (5.4) does not imply (5.8). Therefore, the inverse of a LTV lossless system might not be lossless (Example 6.3).

3) Transform Domain Characterization of Lossless Filters and Their Inverses: First recall the transform domain description introduced in Section II-B and the tilde operation defined in Section IV-A. Consider the direct form A filter $\mathbf{E}(n, \mathcal{Z})$ in Fig. 1. Suppose we cascade $\tilde{\mathbf{E}}(n, \mathcal{Z})$ after the filter $\mathbf{E}(n, \mathcal{Z})$; the resulting system is

$$
\begin{aligned}
\tilde{\mathbf{E}}(n, \mathcal{Z}) \mathbf{E}(n, \mathcal{Z}) & =\sum_{i, k} \mathcal{Z}^{k} \mathbf{e}_{k}^{\dagger}(n) \mathbf{e}_{i}(n) \mathcal{Z}^{-i} \\
& =\sum_{i, k} \mathbf{e}_{k}^{\dagger}(n+k) \mathbf{e}_{i}(n+k) \mathcal{Z}^{k-i} \\
& =\sum_{k, l} \mathbf{e}_{k}^{\dagger}(n+k) \mathbf{e}_{k+l}(n+k) \mathcal{Z}^{-l}
\end{aligned}
$$

If the system $\mathbf{E}(n, \mathcal{Z})$ is lossless, i.e., the coefficients $\mathbf{e}_{k}(n)$ satisfy (5.4), then (5.9) reduces to the following:

$$
\tilde{\mathbf{E}}(n, \mathcal{Z}) \mathbf{E}(n, \mathcal{Z})=\mathbf{I} \text {. }
$$

Therefore, an LTV filter $\mathbf{E}(n, \mathcal{Z})$ is lossless if and only if (5.10) holds. The beauty of $(5.10)$ is that it directly tells us the inverse filter is $\tilde{\mathbf{E}}(n, \mathcal{Z})$ ! Note that the inverse $\tilde{\mathbf{E}}(n, \mathcal{Z})$ is in direct form $\mathrm{B}$ while $\mathbf{E}(n, \mathcal{Z})$ is in the direct form $\mathrm{A}$. On the other hand, if a lossless filter is in direct form $\mathrm{B}, \mathbf{R}(\mathcal{Z}, n)$, its inverse can be shown to be the direct form A filter $\tilde{\mathbf{R}}(\mathcal{Z}, n)$. Summarizing the result, we conclude that the inverse of a lossless system $\mathcal{H}$ is given by $\tilde{\mathcal{H}}$.

\section{B. Subtle Properties of Inverses of Lossless LTV Systems}

From Theorem 5.2, we know that lossless LTV systems are always invertible. However the uniqueness, invertibility and losslessness properties of the inverse are not guaranteed (Example 1.1). It turns out that these properties are closely related to each other. More precisely, we can prove the following.

Theorem 5.3: Given a lossless LTV system $\mathcal{H}$, let $\mathcal{G}$ be one of its inverses. Then the following are equivalent:

a) $\mathcal{G}$ is lossless;

b) $\mathcal{G}$ is invertible and its unique inverse is $\mathcal{H}$;

c) $\mathcal{H}$ maps $l_{2}(M)$ onto $l_{2}(M)$.

a) Proof that a) implies $b$ ): Let the inverse $\mathcal{G}$ be lossless. By Theorem 5.2, we know that $\mathcal{G}$ is invertible. We need only to prove that the inverse of $\mathcal{G}$ is unique and equal to $\mathcal{H}$. Let $\mathcal{F}$ be any inverse of $\mathcal{G}$, so $\mathcal{F G}=\mathcal{I}$. By definition, $\mathcal{G}$ is an inverse of $\mathcal{H}$, so we also have $\mathcal{G H}=\mathcal{I}$. Premultipling both sides by $\mathcal{F}$, we get $\mathcal{F G H}=\mathcal{F}$. Using the fact $\mathcal{F} \mathcal{G}=\mathcal{I}$ we arrive at $\mathcal{H}=\mathcal{F}$. Thus, the only inverse of $\mathcal{G}$ is $\mathcal{H}$.

b) Proof that b) implies $c$ ): Suppose the mapping $\mathcal{H}$ from $l_{2}(M)$ to $l_{2}(M)$ is not onto. Then, there exists $\mathbf{y}_{0}(n) \in l_{2}(M)$ such that $\mathbf{y}_{0}(n) \neq \mathcal{H} \mathbf{x}(n)$ for any $\mathbf{x}(n) \in l_{2}$. Since $\mathcal{G}$ is invertible, and its unique inverse is $\mathcal{H}$, we have $\mathcal{H G}=\mathcal{I}$. Applying the previous equation to the signal $\mathrm{y}_{0}(n)$, we have $\mathcal{H}\left[\mathcal{G}_{0}(n)\right]=\mathbf{y}_{0}(n)$. That means $\mathbf{y}_{0}(n)$ is in the range of $\mathcal{H}$, a contradiction! Therefore, b) implies c). 
c) Proof that c) implies a): By the onto property, for all possible sequences $\mathbf{y}(n) \in l_{2}$, there exists a sequence $\mathbf{x}(n) \in l_{2}$ such that $\mathcal{H} \mathbf{x}(n)=\mathbf{y}(n)$. Since $\mathcal{H}$ is lossless, we have $\sum_{n} \mathbf{x}^{\dagger}(n) \mathbf{x}(n)=\sum_{n} \mathbf{y}^{\dagger}(n) \mathbf{y}(n)$. However, $\mathcal{G H} \mathbf{x}(n)=\mathcal{G} \mathbf{y}(n)=\mathbf{x}(n)$ (because $\mathcal{G H}=\mathcal{I}$ ). Thus, for all $\mathbf{y}(n) \in l_{2}$, the signal $\mathcal{G} \mathbf{y}(n)$ has the same energy as $\mathbf{y}(n)$. Therefore, $\mathcal{G}$ is lossless.

Corollary 1-Uniqueness of the Inverse: If any of the three conditions in Theorem 5.3 is true, then the inverse $\mathcal{G}$ given in Theorem 5.3 is unique.

Proof: We will prove the condition b) implies that $\mathcal{G}$ is unique. Suppose that there are two different systems $\mathcal{G}_{1}$ and $\mathcal{G}_{2}$, such that $\mathcal{G}_{1} \mathcal{H}=\mathcal{G}_{2} \mathcal{H}=\mathcal{I}$. From b), we are given that $\mathcal{G}_{2}$ has the unique inverse $\mathcal{H}$, i.e., $\mathcal{H G}_{2}=\mathcal{I}$. Premultiplying $\mathcal{G}_{1}$ to the previous equation, we have $\mathcal{G}_{1} \mathcal{H} \mathcal{G}_{2}=\mathcal{G}_{1}$. Using the identity $\mathcal{G}_{1} \mathcal{H}=\mathcal{I}$, we have $\mathcal{G}_{1}=\mathcal{G}_{2}$.

As a consequence of the above theorem and corollary, the losslessness, invertibility, and uniqueness of an inverse of a lossless system are the same. They are equivalent to the completeness of the range of the original system $\mathcal{H}$. If one of the inverses of a lossless system is noninvertible, then all of its inverses are non invertible (hence, nonlossless). Therefore, an invertible inverse lossless (IIL) system always has a unique lossless inverse while a non invertible inverse lossless (NIL) system can never have a lossless inverse. Even though the inverses of a NIL system are not lossless, we will show in the following that there will always exist a unique passive inverse.

Theorem 5.4 Existence of Unique Passive Inverse: Given a NIL system $\mathcal{H}$, there is always a unique passive inverse. The unique passive inverse is given by $\tilde{\mathcal{H}}$.

Proof: We split the proof into three parts: i) The existence of passive inverse; ii) the uniqueness of passive inverse; and iii) the passiveness of $\tilde{\mathcal{H}}$. For part iii), it is sufficient to establish the passiveness of $\tilde{\mathcal{H}}$ since $\tilde{\mathcal{H}}$ is always an inverse of $\mathcal{H}$ (Section $\mathrm{V}-\mathrm{A}-3$ ).

i) Let $\mathcal{H}$ be a NIL system and $\mathcal{G}$ be any inverse of $\mathcal{H}$. Let $\mathcal{R}(\mathcal{H}) \subset l_{2}(M)$ denote the range of $\mathcal{H}$ and $\mathcal{R}^{\perp}(\mathcal{H}) \subset l_{2}(M)$ be the orthogonal complement of $\mathcal{R}(\mathcal{H})$, i.e.,

$$
\begin{gathered}
\mathcal{R}(\mathcal{H})=\left\{\mathbf{y}(n) \in l_{2}(M) \mid \mathbf{y}(n)=\mathcal{H} \mathbf{x}(n)\right. \\
\text { for some } \left.\mathrm{x}(n) \in l_{2}(M)\right\} \\
\mathcal{R}^{\perp}(\mathcal{H})=\left\{\mathbf{y}(n) \in l_{2}(M) \mid\langle\mathcal{H} \mathbf{x}(n), \mathbf{y}(n)\rangle=0\right. \\
\left.\quad \text { for all } \mathbf{x}(n) \in l_{2}(M)\right\}
\end{gathered}
$$

Since $\mathcal{H}$ is a linear system, both $\mathcal{R}(\mathcal{H})$ and $\mathcal{R}^{\perp}(\mathcal{H})$ are subspaces of $l_{2}(M)$ and $\mathcal{R}(\mathcal{H}) \oplus \mathcal{R}^{\perp}(\mathcal{H})=l_{2}(M)$. Since $\mathcal{G}$ is an inverse of $\mathcal{H}$, it maps $\mathcal{R}(\mathcal{H})$ onto $l_{2}$. Let $\mathcal{G}_{0}$ be the following linear system:

$$
\mathcal{G}_{0} \mathbf{y}(n)= \begin{cases}\mathcal{G} \mathbf{y}(n), & \text { for } \mathbf{y}(n) \in \mathcal{R}(\mathcal{H}) \\ 0, & \text { for } \mathbf{y}(n) \in \mathcal{R}^{-}(\mathcal{H})\end{cases}
$$

Clearly, $\mathcal{G}_{0}$ is also an inverse of $\mathcal{H}$. For any $\mathbf{y}(n) \in l_{2}$, there are unique $\mathrm{y}_{0}(n) \in \mathcal{R}^{\perp}(\mathcal{H})$ and $\mathrm{y}_{1}(n) \in \mathcal{R}(\mathcal{H})$ such that $\mathbf{y}(n)=\mathbf{y}_{0}(n)+\mathbf{y}_{1}(n)$. The norm of the vectors satisfies $\|\mathbf{y}(n)\|^{2}=\left\|\mathbf{y}_{0}(n)\right\|^{2}+\left\|\mathbf{y}_{1}(n)\right\|^{2}$ because $\left\langle\mathrm{y}_{0}(n), \mathrm{y}_{1}(n)\right\rangle=0$. Applying $\mathcal{G}_{0}$ to the input $\mathbf{y}(n)$, we have $\mathcal{G}_{0} \mathbf{y}(n)=\mathcal{G}_{1}(n)$. Since $\mathcal{H}$ is lossless, we have $\left\|\mathcal{G}_{0} \mathbf{y}(n)\right\|^{2}=\left\|\mathcal{G}_{1}(n)\right\|^{2}=\left\|\mathbf{y}_{1}(n)\right\|^{2} \leq$ $\|\mathrm{y}(n)\|^{2}$. Hence, $\mathcal{G}_{0}$ is a passive inverse of $\mathcal{H}$.

ii) To prove the uniqueness of $\mathcal{G}_{0}$, assume that there is another passive inverse $\mathcal{G}_{1} \neq \mathcal{G}_{0}$. Since $\mathcal{G}_{1} \not \equiv \mathcal{G}_{0}$, there is a $\mathrm{y}_{0}(n) \in \mathcal{R}^{\perp}(\mathcal{H})$ such that $\mathrm{x}_{0}(n)=\mathcal{G}_{1} \mathrm{y}_{0}(n) \neq 0$. Let $\mathrm{y}_{1}(n) \in \mathcal{R}(\mathcal{H})$ be a signal such that $\mathrm{x}_{1}(n)=$ $\mathcal{G}_{1} \mathbf{y}_{1}(n)$ and $\left\langle\mathbf{x}_{0}(n), \mathbf{x}_{1}(n)\right\rangle \neq 0$ (this is always possible because $\mathrm{x}_{1}(n)$ can be an arbitrary $l_{2}(M)$ signal and $\mathrm{x}_{0}(n)$ is not identically zero). Consider $\mathbf{x}(n)=\mathcal{G}_{1}\left[\mathbf{y}_{0}(n)+c \mathbf{y}_{1}(n)\right]$. We have

$$
\begin{aligned}
\|\mathbf{x}(n)\|^{2}= & \left\|\mathbf{x}_{0}(n)\right\|^{2}+|c|^{2}\left\|\mathbf{y}_{1}(n)\right\|^{2} \\
& +c \sum_{n}\left[\mathbf{x}_{1}^{\dagger}(n) \mathbf{x}_{0}(n)+\mathbf{x}_{0}^{\dagger}(n) \mathbf{x}_{1}(n)\right]
\end{aligned}
$$

where we have used $\left\|\mathrm{x}_{1}(n)\right\|=\left\|\mathrm{y}_{1}(n)\right\|$, which follows from the fact that $\mathcal{H}$ is lossless. For given $\mathrm{x}_{0}(n)$ and $\mathrm{x}_{1}(n)$, one can always find a constant $c$ such that $c \sum_{n}\left[\mathbf{x}_{1}^{\dagger}(n) \mathbf{x}_{0}(n)+\mathbf{x}_{0}^{\dagger}(n) \mathbf{x}_{1}(n)\right]>\left\|\mathbf{y}_{0}(n)\right\|^{2}$, which implies that $\mathcal{G}_{1}$ is not passive, a contradiction! Therefore, $\mathcal{G}_{0}$ is the only passive inverse.

iii) We will prove this for the case where $\mathcal{H}$ is in direct form $A$. The proof for the direct form $B$ is similar. Assume that the NIL system $\mathcal{H}$ is $\mathbf{E}(n, \mathcal{Z})=$ $\sum_{k} \mathrm{e}_{k}(n) \mathcal{Z}^{-k}$. We only need to establish the passiveness of $\hat{\mathbf{E}}(n, \mathcal{Z})=\sum_{k} \mathcal{Z}^{k} \mathbf{e}_{k}^{\dagger}(n)$; i.e., we need to prove $\tilde{\mathbf{E}}(n, \mathcal{Z}) \mathbf{y}(n)=0$ for all $\mathbf{y}(n) \in \mathcal{R}^{\perp}(\mathbf{E})$. From $(5.11 \mathrm{~b})$, we have

$$
\begin{gathered}
\mathcal{R}^{\perp}(\mathbf{E})=\left\{\mathbf{y}(n) \in l_{2}(M) \mid\langle\mathbf{E}(n, \mathcal{Z}) \mathbf{x}(n), \mathbf{y}(n)\rangle=0\right. \\
\text { for all } \left.\mathbf{x}(n) \in l_{2}(M)\right\} .
\end{gathered}
$$

That means, for all $\mathrm{y}(n) \in \mathcal{R}^{\perp}(\mathrm{E})$, we have

$$
\begin{gathered}
\sum_{n}\left[\sum_{k} \mathrm{e}_{k}(n) \mathrm{x}(n-k)\right]^{\dagger} \mathrm{y}(n) \\
=0, \quad \text { for all } \mathrm{x}(n) \in l_{2}(M) .
\end{gathered}
$$

After some simplifications, we get

$$
\begin{aligned}
& \sum_{n} \mathrm{x}^{\dagger}(n)\left[\sum_{k} \mathbf{e}_{k}^{\dagger}(n+k) \mathbf{y}(n+k)\right] \\
& \quad=0, \quad \text { for all } \mathbf{x}(n) \in l_{2}(M), \mathbf{y}(n) \in \mathcal{R}^{\perp}(\mathbf{E})
\end{aligned}
$$

Since $\mathrm{x}(n)$ is an arbitrary $l_{2}(M)$ signal, we conclude that $\tilde{\mathbb{E}}(n, \mathcal{Z}) \mathbf{y}(n)=\sum_{k} \mathrm{e}_{k}^{\dagger}(n+k) \mathbf{y}(n+k)=0$. The proof is complete.

\section{Lossless Time-Varying Filter Banks}

In Section IV, we have demonstrated the usefulness of the transform domain description in analyzing the TVFB's. In this subsection, we will study the lossless TVFB's in the transform domain. To relate all the theory developed so far in this section to the TVFB's, we use the polyphase representation. 
1) TVFB with Lossless Analysis Bank: Consider Fig. 12. Let $\mathbf{E}(M n, \mathcal{Z})$ be lossless. If we take the analysis and synthesis polyphase matrices as $\mathbf{E}(M n, \mathcal{Z})$ and $\tilde{\mathbf{E}}(M n, \mathcal{Z})$, respectively, then we have a PR TVFB whose analysis bank is lossless. Using (4.4) and (4.9), one can show in this case that the analysis and the synthesis filters are related as

$$
\begin{aligned}
F^{k}(\mathcal{Z}, M n) & =\tilde{H}^{k}(M n, \mathcal{Z}) \\
f_{i}^{k}(M n) & =h_{-i}^{* k}(M n), \quad \forall n .
\end{aligned}
$$

Therefore, the coefficients of the synthesis filters $f_{i}^{k}(M n)$ are the mirror-image conjugates of the coefficients of the analysis filters $h_{i}^{k}(M n)$. As we explained in Section IV, only the coefficients at time $M n$ are relevant to the TVFB.

2) IIL TVFB: If the synthesis polyphase matrix $\mathbf{R}(\mathcal{Z}, M n)$ is invertible (i.e., the TVFB is an IIL system), then we have $\tilde{\mathbf{R}}(\mathcal{Z}, M n) \mathbf{R}(\mathcal{Z}, M n)=\mathbf{I}$. This implies that the synthesis filters satisfy the following relation:

$$
\left[\tilde{F}^{k}(n, \mathcal{Z}) F^{l}(n, \mathcal{Z})\right]_{\downarrow M}=\delta(k-l)
$$

where the notation $[\bullet]_{\downarrow M}$ is given in (3.7). The above equation can be viewed as a time-varying generalization of the orthonormality condition of the synthesis filters defined in [3], [13], [19], [20]. Note that if the analysis bank is an IIL system, the synthesis bank that yields PR is clearly also an IIL system. Therefore, we can call such an FB an IIL TVFB.

\section{Remarks:}

1) As we have known, the NIL systems do not have lossless inverse. So we might think that this may result in a coding gain, which is smaller than 1 . However, because of Theorem 5.4, there is always a passive inverse for any lossless analysis system. If the passive inverse is used in the reconstruction, the noise introduced in the subband will not be amplified. Combining this with the fact that the analysis bank is lossless, we conclude that the coding gain $\geq 1$.

2) It should be mentioned that if the inverse of a LTV system is lossless, then the LTV system itself is lossless. Therefore, there is no nonlossless system with lossless inverse, even in the LTV case.

\section{EXAMPLES OF LOSSLESS LTV SYSTEMS}

In this section, we will provide three lossless LTV examples. The first two examples are IIL systems while the third example is an NIL system.

1) Example 6.1-A Nontrivial SISO FIR IIL System: Consider the following LPTV system $\mathcal{H}_{1}: y(n)=h_{-1}(n) x(n+1)+$ $h_{0}(n) x(n)+h_{1}(n) x(n-1)$ where the coefficients are

$$
\begin{aligned}
& h_{-1}(n)= \begin{cases}0, & \text { for } n \text { even } \\
\frac{1}{\sqrt{2}}, & \text { for } n \text { odd }\end{cases} \\
& h_{0}(n)= \begin{cases}\frac{1}{\sqrt{2}}, & \text { for } n \text { even } \\
\frac{-1}{\sqrt{2}}, & \text { for } n \text { odd }\end{cases}
\end{aligned}
$$

$$
h_{1}(n)= \begin{cases}\frac{1}{\sqrt{2}}, & \text { for } n \text { even } \\ 0, & \text { for } n \text { odd }\end{cases}
$$

The output of the above system is

$$
y(n)= \begin{cases}\frac{x(n)+x(n-1)}{\sqrt{2}} & \text { for } n \text { even } \\ \frac{x(n+1)-x(n)}{\sqrt{2}} & \text { for } n \text { odd. }\end{cases}
$$

One can verify that the coefficients defined in (6.1) satisfy the lossless condition (5.4). As a consistency check, we can verify the output energy $\sum_{n}|y(n)|^{2}=\sum_{n}|x(n)|^{2}$. This example shows that there exist nontrivial scalar FIR lossless systems in the LTV case. The inverse of $\mathcal{H}_{1}$ is given by $\mathcal{G}_{1}: \hat{x}(n)=$ $h_{-1}^{*}(n-1) y(n-1)+h_{0}^{*}(n) y(n)+h_{1}^{*}(n+1) y(n+1)$, where the coefficients $h_{i}(n)$ are the same as (6.1). The inverse of the scalar lossless FIR system is also FIR! This is impossible in the LTI case except for the trivial system of the form $e^{j \theta} z^{-k}$. Furthermore, one can verify that $\mathcal{G}_{1} \equiv \mathcal{H}_{1}$. We have a system that is its own inverse! Therefore, the inverse $\mathcal{G}_{1}$ is also lossless, which implies that $\mathcal{H}_{1}$ is an IIL system. Since $M=1$, the analysis and synthesis filters are themselves the polyphase matrices $\mathcal{H}_{1}, \mathcal{G}_{1}$, respectively.

2) Example 6.2-An $M \times M$ IIL System: Consider the following first-order system $\mathcal{H}_{2}$ :

$$
\mathbf{y}(n)=\left[\mathbf{I}-\mathbf{v}(n) \mathbf{v}^{\dagger}(n)\right] \mathbf{x}(n)+\mathbf{v}(n) \mathbf{v}^{\dagger}(n-1) \mathbf{x}(n-1)
$$

where the $M \times 1$ vector $\mathbf{v}(n)$ satisfies $\mathbf{v}^{\dagger}(n) \mathbf{v}(n)=1$ for all $n$. The system coefficients are $\mathbf{e}_{0}(n)=\left[\mathbf{I}-\mathbf{v}(n) \mathbf{v}^{\dagger}(n)\right]$ and $\mathbf{e}_{1}(n)=\mathbf{v}(n) \mathbf{v}^{\dagger}(n-1)$. These coefficients have the properties that $\mathbf{e}_{0}^{\dagger}(n) \mathbf{e}_{1}(n)=\mathbf{e}_{1}^{\dagger}(n) \mathbf{e}_{0}(n)=\mathbf{0}$ and $\mathbf{e}_{0}^{\dagger}(n) \mathbf{e}_{0}(n)=\mathbf{e}_{0}(n)$. By using these properties, one can verify that the coefficients satisfy (5.4). Hence, the system $\mathcal{H}_{2}$ in (6.3) is lossless. Its inverse is given as $\mathcal{G}_{2}$ :

$$
\hat{\mathbf{x}}(n)=\left[\mathbf{I}-\mathbf{v}(n) \mathbf{v}^{\dagger}(n)\right] \mathbf{y}(n)+\mathbf{v}(n) \mathbf{v}^{\dagger}(n+1) \mathbf{y}(n+1) .
$$

One can show by directly substituting the coefficients into (5.8) that the inverse $\mathcal{G}_{2}$ defined in (6.4) is also lossless. Therefore, $\mathcal{H}_{2}$ is an IIL system. The analysis filters are given as

$$
\begin{aligned}
H^{k}(M n, \mathcal{Z})= & {\left[1-\left|v_{k}(n)\right|^{2}\right] \mathcal{Z}^{-k}-\sum_{i \neq k} v_{k}(n) v_{i}^{*}(n) \mathcal{Z}^{-i} } \\
& +\sum_{i=0}^{M-1} v_{k}(n) v_{i}^{*}(n-1) \mathcal{Z}^{-M-i}
\end{aligned}
$$

where $v_{k}(n)$ is the $k$ th element of the vector $\mathbf{v}(n)$. The synthesis filters are $F^{k}(\mathcal{Z}, M n)=\tilde{H}^{k}(M n, \mathcal{Z})$.

3) Example 6.3.-An $M \times M$ NIL System: Consider the following LTV system $\mathcal{H}_{3}$ : The system equation is the same as (6.3) but the vectors are chosen as $\mathbf{v}(n)=0$ for $n<0$ and $\mathbf{v}(n)=$ arbitrary unit norm vector for $n \geq 0$. The output of the system can be described as

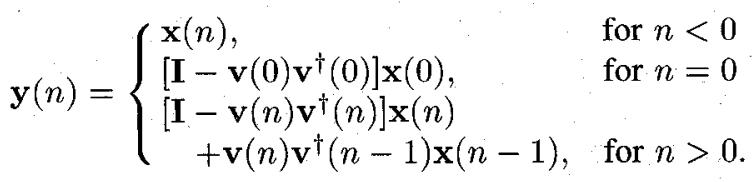


One can verify that the system $\mathcal{H}_{3}$ described above satisfies (5.4). Hence, it is lossless. From the above equation, we know that the output $\mathbf{y}(0)$ is always in the range of the matrix $\left[\mathbf{I}-\mathbf{v}(0) \mathbf{v}^{\dagger}(0)\right]$. Since the matrix $\left[\mathbf{I}-\mathbf{v}(0) \mathbf{v}^{\dagger}(0)\right]$ with unit norm vector $\mathbf{v}(0)$ is always singular, the output $\mathbf{y}(0)$, therefore, cannot be arbitrary. Therefore, the lossless system $\mathcal{H}_{3}$ does not map $l_{2}(M)$ onto $l_{2}(M)$. Theorem 5.3 implies that its inverse is not lossless (hence, not invertible). Thus, $\mathcal{H}_{3}$ is a NIL system. Its unique passive inverse $\mathcal{G}_{3}$ is given as follows:

$$
\hat{\mathbf{x}}(n)= \begin{cases}\mathbf{y}(n), & \text { for } n<0 \\
{\left[\begin{array}{l}
\left.\mathbf{I}-\mathbf{v}(n) \mathbf{v}^{\dagger}(n)\right] \mathbf{y}(n) \\
+\mathbf{v}(n) \mathbf{v}^{\dagger}(n+1) \mathbf{y}(n+1),
\end{array}\right.} & \text { for } n \geq 0\end{cases}
$$

The corresponding analysis filters are given by

$$
\begin{aligned}
& H^{k}(M n, \mathcal{Z}) \\
& \quad \begin{cases}\mathcal{Z}^{-k}, & \text { for } n<0 \\
\left(1-\left|v_{k}(0)\right|^{2}\right) \mathcal{Z}^{-k} & \text { for } n=0 \\
-\sum_{i \neq k} v_{k}(0) v_{i}^{*}(0) \mathcal{Z}^{-i}, & \\
\left(1-\left|v_{k}(n)\right|^{2}\right) \mathcal{Z}^{-k}-\sum_{i \neq k} v_{k}(n) v_{i}^{*}(n) \mathcal{Z}^{-i} & \\
+\sum_{i=0}^{M-1} v_{k}(n) v_{i}^{*}(n-1) \mathcal{Z}^{-M-i}, & \text { for } n>0 .\end{cases}
\end{aligned}
$$

The unique passive synthesis bank is given as $F^{k}(\mathcal{Z}, M n)=$ $\tilde{H}^{k}(M n, \mathcal{Z})$.

Comments:

1) One can verify that the lossless system $\mathcal{H}_{1}$ in Example 6.1 can be obtained by unblocking (see Section III-C) a $2 \times 2$ LTI system with transfer matrix $(1 / \sqrt{2})\left[\begin{array}{ll}1 & 1 \\ 1 & -1\end{array}\right]$. Since $\mathbf{T}^{-1}=\mathbf{T}$, the inverse of $\mathcal{H}_{1}$, which the unblocked version of $\mathbf{T}^{-1}$, is identical to $\mathcal{H}_{1}$ itself!

2) The lossless system $\mathcal{H}_{2}$ in Example 6.2 can be viewed as the generalization of the LTI degree-one building block studied in ch. 14 of [12]. It is shown in [10] that in some cases, $\mathcal{H}_{2}$ can be used as a building block to factorize higher order lossless systems.

\section{Discrete-Time Frames And Riesz Bases for $l_{2}$}

In [14], the authors introduced a vector space framework for LTI FB's. It was shown how the vector space manipulation simplifies the analysis of conventional LTI FB theory. In particular, it is true that the synthesis functions of a PR FB form a discrete-time Riesz basis for $l_{2}$. This result was also shown in [13] by using a different approach. In the case of TVFB's, we will show that a PR TVFB in general will only give rise to a discrete-time frame. If, in addition to being PR, the synthesis polyphase matrix is also invertible, in this case, the TVFB will generate a Riesz basis. In the lossless case, a NIL PR TVFB produces a tight frame with a unity frame bound while an IL PR TVFB will generate an orthonormal basis. Tutorials on frames and $l_{2}$ bases can be found in [17]- [20].

\section{A. Frames and Noninvertible Inverse LTV Systems}

Recall from Section IV that if a TVFB has PR, then any $l_{2}$ signal $x(n)$ can be expressed in terms of the filter outputs as in (4.2). If we define the families of the double index functions $\eta_{k m}(n)$ and $\theta_{k m}(n)$, respectively, as

$$
\begin{aligned}
& \eta_{k m}(n)=f_{n-M m}^{k}(M m), \\
& \theta_{k m}^{*}(n)=h_{M m-n}^{k}(M m)
\end{aligned}
$$

where $0 \leq k \leq M-1$ and $-\infty \leq m \leq \infty$; then, (4.2) can be rewritten as

$$
x(n)=\sum_{k=0}^{M-1} \sum_{m} y_{k}(m) \eta_{k m}(n)
$$

where

$$
\begin{aligned}
y_{k}(m) & =\left\langle x(n), \theta_{k m}(n)\right\rangle \\
& =\sum_{n} x(n) \theta_{k m}^{*}(n) .
\end{aligned}
$$

The functions $\eta_{k m}(n)$ and $\theta_{k m}(n)$ are, respectively, called the synthesis and analysis functions in [14]. From (7.1), the synthesis functions $\eta_{k m}(n)$ are the synthesis filter coefficients at time $M m$ shifted to the right by $M m$. In the LTI case, both $h_{i}^{k}(n)$ and $f_{i}^{k}(n)$ are independent of $n$. All the analysis and synthesis functions, $\theta_{k m}(n)$ and $\eta_{k m}(n)$ for a fixed $k$ are, respectively, shifted versions of $\theta_{k 0}(n)$ and $\eta_{k 0}(n)$. That means for all $m, \theta_{k m}(n)$ and $\eta_{k m}(n)$ have the same shape as $\theta_{k 0}(n)$ and $\eta_{k 0}(n)$, respectively. In the LTV case, this property no longer holds as the filters are time varying. If the analysis bank is lossless, recall that one of the possible set of synthesis filters (in general the synthesis filters are not unique; see Section V-B) for PR is given in (5.17). If the synthesis filters are chosen so, the synthesis bank is passive. Using (5.17) in (7.1), we have

$$
\theta_{k m}(n)=\eta_{k m}(n)
$$

The analysis functions are identical to the synthesis functions. In the following, we will provide an example that shows that the synthesis (or the analysis) functions corresponding to a PR TVFB are, in general, not independent (hence, cannot be a discrete-time basis for $l_{2}$ ).

1) Example 7.1.-Linear Dependency of Analysis/Synthesis Functions: Consider the PR TVFB given in Example 6.3. For simplicity we choose $M=2$ and $\mathbf{v}(n)=\left[\begin{array}{ll}1 / \sqrt{2} & 1 / \sqrt{2}\end{array}\right]^{T}$ for $n \geq 0$-in this case, $k=0$ or 1 . The analysis bank is a NIL system; hence, the synthesis bank is not invertible. The analysis functions are given as

$$
\begin{aligned}
& \theta_{0 m}(n) \\
& = \begin{cases}\delta(n-2 m), & \text { for } m<0 \\
0.5 \delta(n)-0.5 \delta(n+1), & \text { for } m=0 \\
0.5 \delta(n-2 m)-0.5 \delta(n+1-2 m) & \\
+0.5 \delta(n+2-2 m) & \\
+0.5 \delta(n+3-2 m), & \text { for } m>0\end{cases}
\end{aligned}
$$




$$
\begin{aligned}
& \theta_{1 m}(n) \\
& = \begin{cases}\delta(n+1-2 m), & \text { for } m<0 \\
-0.5 \delta(n)+0.5 \delta(n+1), & \text { for } m=0 \\
-0.5 \delta(n-2 m)+0.5 \delta(n+1-2 m) & \\
+0.5 \delta(n+2-2 m) & \\
+0.5 \delta(n+3-2 m), & \text { for } m>0 .\end{cases}
\end{aligned}
$$

One set of synthesis functions is given as $\eta_{k m}(n)=\theta_{k m}(n)$ since the analysis bank is lossless. In this case, one can verify that

$$
\theta_{00}(n)-\theta_{01}(n)-\theta_{10}(n)+\theta_{11}(n)=0, \quad \forall n .
$$

Therefore, the analysis (or synthesis) functions are linearly dependent.

From the above example, we have seen that the analysis (or synthesis) functions of a PR TVFB does not form a basis. However, we can show the following frame property:

Theorem 7.1. Discrete-Time Frame for $l_{2}$ : The synthesis functions $\eta_{k m}(n)$ of a PR FIR TVFB form a compactly supported discrete-time frame for $l_{2}$. The dual frame is given by the analysis functions $\theta_{k m}(n)$. Moreover, if the analysis bank is lossless, then the functions $\eta_{k m}(n)=\theta_{k m}(n)$ form a tight frame with unity frame bound.

Proof: If the TVFB is FIR, the functions $\eta_{k m}(n)$ and $\theta_{k m}(n)$ clearly have compact support. It is shown in Appendix A that for any $x(n) \in l_{2}$, there exist $B_{1}<\infty$ and $B_{2}<\infty$ such that

$$
\begin{aligned}
& \sum_{k, m}\left|\left\langle x(n), \eta_{k m}(n)\right\rangle\right|^{2} \leq B_{1}\|x(n)\|^{2} \\
& \sum_{k, m}\left|\left\langle x(n), \theta_{k m}(n)\right\rangle\right|^{2} \leq B_{2}\|x(n)\|^{2}
\end{aligned}
$$

Notice that the index $k$ runs from 0 to $M-1$ only. From (7.2), we have

$$
\begin{aligned}
\|x(n)\|^{2} \leq & \sum_{k, m}\left|\left\langle x(n), \theta_{k m}(n)\right\rangle\right|\left|\left\langle\eta_{k m}(n), x(n)\right\rangle\right| \\
\leq & {\left[\sum_{k, m}\left|\left\langle x(n), \theta_{k m}(n)\right\rangle\right|^{2}\right]^{1 / 2} } \\
& \cdot\left[\sum_{k, m}\left|\left\langle x(n), \eta_{k m}(n)\right\rangle\right|^{2}\right]^{1 / 2}
\end{aligned}
$$

where we have used the Cauchy-Schwartz inequality. Substituting (7.6b) into (7.7), we have shown that there exist $A_{1}=1 / B_{2}>0$ and $B_{1}<\infty$ such that

$$
\begin{aligned}
A_{1}\|x(n)\|^{2} & \leq \sum_{k, m}\left|\left\langle x(n), \eta_{k m}(n)\right\rangle\right|^{2} \\
& \leq B_{1}\|x(n)\|^{2}
\end{aligned}
$$

for all $x(n) \in l_{2}$. Therefore, the synthesis functions $\eta_{k m}(n)$ form a frame [17]-[20]. Its dual frame is given by $\theta_{k m}(n)$. In the case when the analysis bank is lossless, we have

$$
\begin{aligned}
\|x(n)\|^{2} & =\sum_{k, m}\left|y_{k}(m)\right|^{2} \\
& =\sum_{k, m}\left|\left\langle x(n), \theta_{k m}(n)\right\rangle\right|^{2}
\end{aligned}
$$

which shows that $A_{1}=B_{1}=1$, i.e., the functions $\theta_{k m}(n)$ form a tight frame with unity frame bound.

\section{Remarks:}

1) Given a frame $\eta_{k m}(n)$ for $l_{2}$, where $-\infty<m<\infty$ and $0 \leq k \leq M-1$, there is always a dual frame $\theta_{k m}(n)$. One can always construct an underlying $M$-channel PR TVFB by choosing the analysis and synthesis filters as in (7.1). Then we can get a stable PR TVFB. In addition, if both the frame and the dual frame have compact support, it is clear that the filters have only finitely many nonzero coefficients.

2) Equation (7.9) can be viewed as the Paserval relation which states the energy preservation.

3) The reason why $\eta_{k m}(n)$ might fail to be a Riesz basis is because the functions may no longer be independent as demonstrated in Example 7.1. The proof of independence in [14] does not go through as $\eta_{k \dot{m}}(n)$ are no longer shifted versions of $\eta_{k 0}(n)$.

\section{B. Bases and Invertible Inverse LTV Systems}

Recall that if the synthesis bank of a PR TVFB is also invertible, the filters satisfy the condition in (4.6). By using the definition of the analysis and synthesis functions in (7.1), we can show by directly expanding (4.6) that

$$
\left\langle\eta_{k_{0} m_{0}}(n), \theta_{k_{1} m_{1}}(n)\right\rangle=\delta\left(k_{0}-k_{1}\right) \delta\left(m_{0}-m_{1}\right)
$$

Therefore the analysis and the synthesis functions satisfy the biorthogonality condition. In the case of IIL TVFB's, we have $\eta_{k m}(n)=\theta_{k m}(n)$. Equation (7.10) reduces to the following orthonormality condition:

$$
\left\langle\eta_{k_{0} m_{o}}(n), \eta_{k_{1} m_{1}}(n)\right\rangle=\delta\left(k_{0}-k_{1}\right) \delta\left(m_{0}-m_{1}\right)
$$

In this case, we can show the following:

Theorem 7.2. Discrete-Time Basis for $l_{2}$ : Given a stable PR FIR TVFB with an invertible synthesis bank, the corresponding synthesis functions $\eta_{k m}(n)$ form a compactly supported discrete-time Riesz basis for $l_{2}$. The dual basis is given by the analysis functions $\theta_{k m}(n)$. Moreover, if the TVFB is an IIL system, then the functions $\theta_{k m}(n)=\eta_{k m}(n)$ form an orthonormal basis for $l_{2}$.

Proof: Because of Theorem 7.1, we need only to establish the independence of the synthesis functions $\eta_{k m}(n)$. The independence of $\eta_{k m}(n)$ follows directly from the biorthogonality condition (7.10). In the case of IIL TVFB, the synthesis functions $\eta_{k m}(n)$ satisfy $\left\|\eta_{k m}(n)\right\|^{2}=1$ [from (7.11)]. By using the fact that a normalized tight frame is an orthonormal basis [19], [20], we have completed the proof. 
Remark: A PR TVFB in general does not form a biorthogonal system. Therefore, a PR TVFB cannot be called a biorthogonal TVFB unless its synthesis bank is invertible. The same is true for a lossless TVFB.

\section{CONCLUSIONS}

In this paper, we have introduced a time-varying polyphase approach to study some basic properties of TVFB's. By using the proposed method, the theory of time-varying multirate signal processing and filter banks can be developed in analogy to the conventional LTI FB's (Sections III and IV). Even though TVFB's share many properties with the LTI FB's, there are some major differences as pointed out in the abstract. We have studied in detail the class of the lossless TVFB's (Section V). Both the time-domain and frequencydomain characterizations of lossless LTV systems are given ((5.4) and (5.10), respectively). We showed that all lossless LTV systems are invertible and its inverse is FIR provided that the original lossless system is FIR (Theorem 5.2). We also showed that the inverse of a lossless LTV system may not be lossless (Examples 1.1 and 6.3). The losslessness, invertibility, uniqueness, and passivity of the inverse are related as in Theorems 5.3 and 5.4. We have demonstrated that the synthesis (or analysis) functions of a PR TVFB may not generate a basis for $l_{2}$ (Example 7.1). These functions, however, form a frame for $l_{2}$ (Theorem 7.1) and become a basis for $l_{2}$ only if the synthesis polyphase matrix is invertible (Theorem 7.2). Moreover, the basis is orthonormal if the TVFB is an IL system. In a companion paper [10], we will show that the time-domain characterization of lossless systems in (5.4) is useful for the parameterization and factorization of lossless LTV systems.

\section{APPENDIX \\ PROOF OF (7.6)}

Since the filters are stable, we have $\sum_{i}\left|f_{i}^{k}(n)\right|<\infty$ and $\sum_{i}\left|h_{i}^{k}(n)\right|<\infty$, for all $k$ and $n$. This, in particular, implies that there is a $B<\infty$ such that $\max _{n}\left|f_{i}^{k}(n)\right| \leq B$ for all $i$ and $k$. Since the filters are FIR, there is a $N<\infty$ such that $f_{i}^{k}(n)=0$ and $h_{i}^{k}(n)=0$ for all $i>N$. By using (7.1) and making a change of variable, we can rewrite the left hand side of (7.6a) as

$$
\begin{aligned}
& \sum_{k=0}^{M-1} \sum_{i=0}^{N-1} \sum_{m}\left|f_{i}^{k}(M m) x(M m+i)\right|^{2} \\
& \quad \leq\|x(n)\|^{2} \sum_{k=0}^{M-1} \sum_{i=0}^{N-1}\left[\max _{m}\left|f_{i}^{k}(M m)\right|\right] \\
& \quad \leq N \cdot B\|x(n)\|^{2} .
\end{aligned}
$$

By taking $B_{1}=N B$, we have proved (7.6a). Similarly, we can prove (7.6b).

\section{REFERENCES}

[1] J. S. Prater and C. M. Loeffler, "Analysis and design of periodically time-varying IIR filters, with applications to transmultiplexing," IEEE Trans. Signal Processing, vol. 40, no. 11, pp. 2715-2725, Nov. 1992.

[2] R. E. Crochiere and L. R. Rabiner, Multirate Digital Signal Processing. Englewood Cliffs, NJ: Prentice-Hall. 1983.

[3] C. Herley, J. Kovacevic, K. Ramachandran, and M. Vetterli, "Tilings of the time-frequency plane: Construction of arbitrary orthogonal bases and fast tiling algorithm," IEEE Trans. Signal Processing, vol. 41, no. 12, pp. 3341-3359, Dec. 1993.

[4] M. J. T. Smith and W. C.-L. Chung, "Recursive time-varying filter banks for subband image coding," IEEE Trans. Image Processing, vol. 4, pp. $885-895$, July 1995 .

[5] J. L. Arrowood, Jr., and M. J. T. Smith, "Exact reconstruction analysis/synthesis filter banks with time-varying filters," in Proc. Int. Conf. Acoust., Speech, Signal Processing, Minneapolis, MN, Apr. 1993, pp. $233-236$

[6] K. Nayebi, M. J. T. Smith, and T. P. Barnwell, "Analysis-synthesis systems based on time-yarying filter banks," in Proc. Int. Conf. Acoust. Speech, Signal Processing, San Francisco, CA, Mar. 1992, pp. 617-620.

[7] C. Herley, "Boundary filters for finite length signals and time-varying filter banks," IEEE Trans. Circuits Syst., vol. 42, pp. 102-114, Feb. 1995

[8] R. A. Gopinath, and C. S. Burrus, "Factorization approach to unitary time-varying filter bank trees and wavelets,". IEEE Trans. Signal Processing, vol. 43, no. 3, pp. 666-680, Mar. 1995.

[9] R. L. de Queiroz and K. R. Rao, "Time-varying lapped transform and wavelet packets," IEEE Trans. Signal Processing, vol. 41, no. 12, pp. 3293-3305, Dec. 1993

[10] S.-M. Phoong and P. P. Vaidyanathan, "Factorizability of lossless time-varying filter banks," Trans. Signal Processing, submitted for publication.

[11] I. Sodagar, K. Nayebi and T. P. Barnwell, "Time-varying filter banks and wavelets," IEEE Trans. Signal Processing, vol. 42, no. 11, pp. 3293-3305, Nov. 1994.

[12] P. P. Vaidyanathan, Multirate Systems and Filter Banks. Englewood Cliffs, NJ: Prentice-Hall, 1993.

[13] I. Djokovic and P. P. Vaidyanathan, "Results on biorthogonal filter banks," Appl. and Computat. Harmon. Anal., vol. 1, pp: 329-343, 1994.

[14] T. Chen and P. P. Vaidyanathan, "Vector space framework for unnification of one- and multidimensional filter bank theory," IEEE Trans. Signal Processing, vol. 42, no. 8, pp. 2006-2022, Aug. 1994.

[15] C. S. Burrus, "Block implementation of digital filters," IEEE Trans. Ciruit Theory, vol. CT-18, pp. 697-701, Nov. 1971.

[16] M. Vetterli, "Perfect transmultiplexers," in Proc. IEEE Int. Conf. Acoust., Speech, Signal Processing, Tokyo, Japan, Apr. 1986, pp. 2567-2570.

[17] R. M. Young, An Introduction to Nonharmonic Fourier Series. New York: Academic, 1980.

[18] C. E. Heil and D. F. Walnut, "Continuous and discrete wavelet transforms," SIAM Rev., vol. 31, p. 628-666, Dec. 1989.

[19] I. Daubechies, Ten Lectures on Wavelets. Philadelphia, PA: SIAM, 1992.

[20] P. P. Vaidyanathan and I. Djokovic, Wavelet Transforms, in Circuits and Filters Handbook, W. K. Chen, Ed. Boca Raton, FL: CRC, 1995.

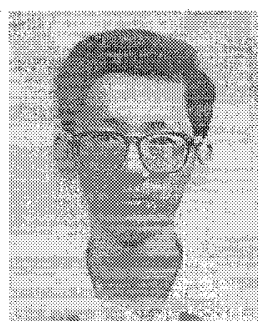

See-May Phoong (S'93) was born in Johor, Malaysia, in 1968. He received the B.S. degree in electrical engineering from National Taiwan, University, Taipei, in 1991 and the M.S. and Ph.D. degrees in electrical engineering from the California Institute of Technology, Pasadena, in 1992 and 1996, respectively

He is currently a member of the faculty of the Department of Electrical and Electronic Engineering, Nanyang Technological University, Singapore. His interests include digital signal processing, multirate filter banks, and wavelet transforms. 


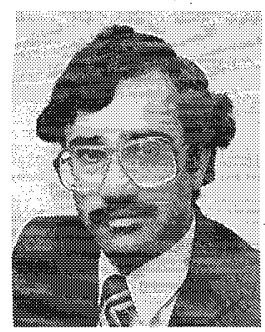

P. P. Vaidyanathan (S'80-M'83-SM'88-F'91) was born in Calcutta, India, on October 16, 1954. He received the B.Sc. degree (with Honors) in physics and the B.Tech and M.Tech degrees in radiophysics and electronics, all from the University of Calcutta, India, in 1974, 1977, and 1979, respectively. He received the Ph.D. degree in electrical and computer engineering from the University of California, Santa Barbara, in 1982 . He was a postdoctoral fellow at the University of California from September 1982 to March 1983.

In March 1983, he joined the Electrical Engineering Department of the California Institute of Technology (Caltech), as an Assistant Professor. Since 1993, he has been Professor of electrical engineering at Caltech. His main research interests are in digital signal processing, multirate systems, wavelet transforms, and adaptive filtering. He is the author of Multirate Systems and Filter Banks, and has written several chapters for signal processing handbooks. He has authored numerous papers for IEEE journals.

Dr. Vaidyanathan served as Vice-Chairman of the Technical Program Committee for the 1983 IEEE International Symposium on Circuits and Systems, and as the Technical Program Chairman for the 1992 IEEE International Symposium on Circuits and Systems. He was an Associate Editor for the IEEE TRANSACTIONS ON CiRCuITS AND SYSTEMS from 1987 to 1987 , and is currently an associate editor IEEE Signal Processing LETTERS. He is a consulting editor for APPLIED AND COMPUTATIONAL HARMONIC ANALYSIS. He received the Award for Excellence in Teaching at Caltech for the years 1983-1984, 1992-1993, and 1993-1994. He also received the NSF's Presidential Young Investigator Award in 1986. In 1989, he recieved the IEEE Acoustics, Speech, and Signal Processing Award for this paper on multirate perfect reconstruction filter banks. In 1990, he was recipient of the S. K. Mitra Memorial Award from the Institute of Electronics and Telecommunications Engineers, India, for joint paper in the IETE JOURNAL He also received the 1995 F. E. Terman Award of the American Society for Engineering Education sponsored by Hewlett Packard Co. He was chosen Distinguished Lecturer for the IEEE Signal Processing Society for the year 1996-1997. He coauthored a paper on linear-phase perfect reconstruction filter banks in the IEEE TRANSACTIONS ON SIGNAL PROCESSING, for which the firs author, T. Nguyen, received the Young Outstanding Author Award in 1993. 